\title{
Rapid Synthesis of the A-E Fragment of Ciguatoxin CTX3C
}

\section{J. Stephen Clark, ${ }^{* \dagger}$ Joanne Conroy, ${ }^{\dagger}$ and Alexander J. Blake ${ }^{\ddagger}$}

${ }^{\dagger}$ WestCHEM, Department of Chemistry, Joseph Black Building, University of Glasgow, University Avenue, Glasgow G12 8QQ, UK

* School of Chemistry, University of Nottingham, University Park, Nottingham NG7 2RD, UK

\section{Supporting Information}

\section{A-C Fragment 10.}

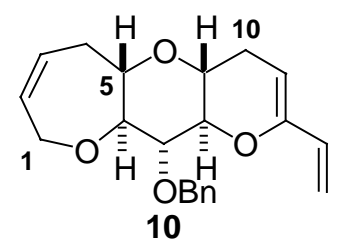

Ethene gas was passed through a solution of the alkynyl ether $\mathbf{8}(80.0 \mathrm{mg}, 0.224 \mathrm{mmol})$ and the ruthenium complex $9(18.0 \mathrm{mg}, 0.0212 \mathrm{mmol})$ in toluene $(30 \mathrm{~mL})$ at $\mathrm{rt}$. After $10 \mathrm{~min}$ the solution was heated to $70{ }^{\circ} \mathrm{C}$ and stirred under a static atmosphere of ethene overnight. The solution mixture was concentrated and the residue was purified by flash column chromatography on silica gel (etherpetrol, 1:9 with $2 \%$ triethylamine), to give the triene $\mathbf{1 0}$ as a colourless solid (44.0 $\mathrm{mg}, 58 \%$ ): $\mathrm{Mp}$ $70-72{ }^{\circ} \mathrm{C} ; \mathrm{R}_{f}=0.26$ (ether-hexane, 1:9); $[\alpha]_{\mathrm{D}}^{23}-71\left(c 1.0, \mathrm{CHCl}_{3}\right) ; v_{\max }\left(\mathrm{CHCl}_{3}\right) 2927,1602 \mathrm{~cm}^{-1}$;

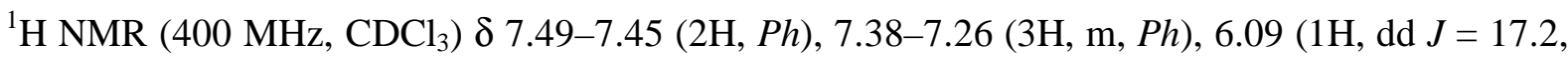
$10.9 \mathrm{~Hz}, \mathrm{H}-13)$ 5.95-5.87 (1H, m, H-2), 5.85-5.77 (1H, m, H-3), 5.50 (1H, d, J=17.2 Hz, H-14), $5.09(1 \mathrm{H}, \mathrm{d}, J=10.9 \mathrm{~Hz}, \mathrm{H}-14), 5.00(1 \mathrm{H}, \mathrm{d}, J=11.4 \mathrm{~Hz}, \mathrm{CH} H \mathrm{Ph}), 4.91(1 \mathrm{H}, \mathrm{d}, J=11.4 \mathrm{~Hz}$, $\mathrm{C} H \mathrm{HPh}), 4.80(1 \mathrm{H}, \mathrm{dd}, J=5.5,2.6 \mathrm{~Hz}, \mathrm{H}-11), 4.34(1 \mathrm{H}, \mathrm{dd}, J=15.6,5.9 \mathrm{~Hz}, \mathrm{H}-1), 4.08(1 \mathrm{H}$, ddd, $J=15.6,5.2,2.6 \mathrm{~Hz}, \mathrm{H}-1), 3.71(1 \mathrm{H}, \mathrm{dd}, J=8.9,8.2 \mathrm{~Hz}, \mathrm{H}-7), 3.63(1 \mathrm{H}, \mathrm{dd}, J=9.2,8.9 \mathrm{~Hz}, \mathrm{H}-8)$, 3.56-3.47 (1H, m, H-9), 3.44 (1H, dd, $J=9.2,8.2 \mathrm{~Hz}, \mathrm{H}-6), 3.37$ (1H, ddd, $J=9.7,9.2,3.9 \mathrm{~Hz}, \mathrm{H}-$ 5), 2.68 (1H, ddd, $J=16.2,7.9,3.9 \mathrm{~Hz}, \mathrm{H}-4), 2.49-2.33$ (2H, m, H-4 and H-10), 2.17 (1H, br dd $J=$ 17.3, 9.6 Hz, H-10); ${ }^{13} \mathrm{C}$ NMR (100 MHz, $\left.\mathrm{CDCl}_{3}\right) \delta 150.6(\mathrm{C}), 139.2(\mathrm{C}), 131.6(\mathrm{CH}), 131.4(\mathrm{CH})$, 128.4 (CH), $128.0(\mathrm{CH}), 127.6(\mathrm{CH}), 127.0(\mathrm{CH}), 113.2\left(\mathrm{CH}_{2}\right), 100.4(\mathrm{CH}), 87.2(\mathrm{CH}), 82.4(\mathrm{CH})$, $78.8(\mathrm{CH}), 76.4(\mathrm{CH}), 75.4\left(\mathrm{CH}_{2}\right), 71.5(\mathrm{CH}), 68.4\left(\mathrm{CH}_{2}\right), 34.7\left(\mathrm{CH}_{2}\right), 28.0\left(\mathrm{CH}_{2}\right)$; LRMS $(\mathrm{CI}) \mathrm{m} / \mathrm{z}$ 358 $\left(\left[\mathrm{M}+\mathrm{NH}_{3}\right]^{+}, 100 \%\right), 342(23) 341\left([\mathrm{M}]^{+}, 97\right)$, 323(19); HRMS (CI, $\left.\mathrm{NH}_{3}\right)$ calcd for $\mathrm{C}_{21} \mathrm{H}_{24} \mathrm{O}_{4}$ $\left[\mathrm{M}^{+}\right]$: 340.1675, found: 340.1683. Found C, 74.08; H, 7.15; $\mathrm{C}_{21} \mathrm{H}_{24} \mathrm{O}_{4}$ requires C, 74.09; H, 7.11. 
Alcohols 16a and 16b.

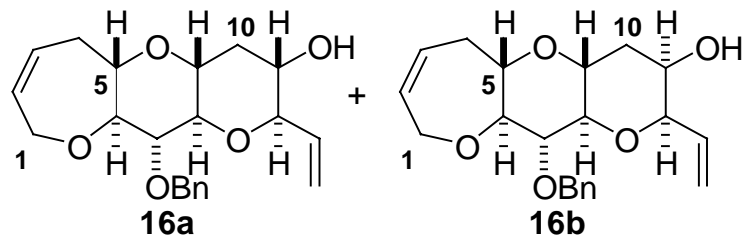

The triene 10 (400 mg, $1.18 \mathrm{mmol})$ was dissolved in $\mathrm{CH}_{2} \mathrm{Cl}_{2}(10 \mathrm{~mL})$ at $0{ }^{\circ} \mathrm{C}$. Dimethyldioxirane $(14.6 \mathrm{~mL}$ of a $\sim 0.08 \mathrm{M}$ in acetone, $1.17 \mathrm{mmol}$ ) was added slowly and the reaction was stirred at $0{ }^{\circ} \mathrm{C}$ for $1 \mathrm{~h}$. The water layer was extracted with $\mathrm{CH}_{2} \mathrm{Cl}_{2}(2 \times 10 \mathrm{~mL})$. The combined organic layers were dried $\left(\mathrm{Na}_{2} \mathrm{SO}_{4}\right)$ and concentrated in vacuo. The crude product was dried under high vacuum (<0.1 mbar) for $10 \mathrm{~min}$ and used without purification.

The crude epoxide was dissolved in dry acetonitrile $(10 \mathrm{~mL})$ under argon and the solution was cooled to $-40{ }^{\circ} \mathrm{C}$. Boron trifluoride etherate $(148 \mu \mathrm{l}, 1.20 \mathrm{mmol})$, and triethylsilane $(561 \mu \mathrm{l}, 3.51$ mmol) were then added and the reaction was stirred for $30 \mathrm{~min}$ and then allowed warm to rt. The reaction was quenched with a sat. aqueous solution of potassium carbonate $(20 \mathrm{~mL})$. The aqueous layer was washed with ether $(2 \times 10 \mathrm{~mL})$ and the combined organic layers were dried $\left(\mathrm{MgSO}_{4}\right)$, filtered and concentrated in vacuo. The product was purified by flash column chromatography on silica gel (petrol-ether, 1:5) to give a mixture of the alcohols 16a and 16b as a colourless solid (298 mg, 71\% over 2 steps). Pure samples of the alcohols 16a and 16b could be obtained by careful chromatography.

16a: Mp 119-120 ${ }^{\circ} \mathrm{C} ; \mathrm{R}_{f}=0.53$ (ether); $[\alpha]_{\mathrm{D}}^{26}+3.2\left(c 1.0, \mathrm{CHCl}_{3}\right) ; v_{\max }\left(\mathrm{CHCl}_{3}\right) 3636,2877 \mathrm{~cm}^{-1}$; ${ }^{1} \mathrm{H}$ NMR (400 MHz, $\left.\mathrm{CDCl}_{3}\right) \delta$ 7.44-7.22 (5H, m, Ph), $5.92(1 \mathrm{H}, \mathrm{ddd}, J=17.3,10.6,6.6 \mathrm{~Hz}, \mathrm{H}-13)$, 5.91-5.84 (1H, m, H-2), 5.82-5.75 (1H, m, H-3), 5.45 (1H, ddd, $J=17.3,1.6,1.3 \mathrm{~Hz}, \mathrm{H}-14), 5.36$ $(1 \mathrm{H}, \mathrm{ddd}, J=10.6,1.6,1.0 \mathrm{~Hz}, \mathrm{H}-14), 4.89$ (1H, d, $J=11.6 \mathrm{~Hz}, \mathrm{CH} H \mathrm{Ph}), 4.82(1 \mathrm{H}, \mathrm{d}, J=11.6, \mathrm{~Hz}$ CHHPh), $4.30(1 \mathrm{H}, \mathrm{dd}, J=15.6,5.9 \mathrm{~Hz}, \mathrm{H}-1), 4.02(1 \mathrm{H}, \mathrm{dd}, J=15.6,5.3,2.6 \mathrm{~Hz}, \mathrm{H}-1), 3.64-3.58$ (1H, m, H-12), 3.54-3.48 (1H, m, H-7), 3.47-3.38 (1H, m, H-11), $3.36(1 \mathrm{H}, \mathrm{dd}, J=9.2,8.0 \mathrm{~Hz}, \mathrm{H}-$ 6), 3.30 (1H, ddd, $J=9.4,9.2,3.8 \mathrm{~Hz}, \mathrm{H}-5), 3.22-3.18$ (2H, m, H-8, H-9), 2.66 (1H, ddd, $J=16.0$, 7.8, 3.8 Hz, H-4), 2.49-2.43 (1H, m, H-10), 2.40-2.29 (1H, m, H-4), 1.83-1.59 (1H, br, OH), 1.61$1.48(1 \mathrm{H}, \mathrm{m}, \mathrm{H}-10) ;{ }^{13} \mathrm{C}$ NMR $\left(100 \mathrm{MHz}, \mathrm{CDCl}_{3}\right) \delta 139.2(\mathrm{C}), 135.3(\mathrm{CH}), 131.4(\mathrm{CH}), 128.2$ $(\mathrm{CH}), 127.8(\mathrm{CH}), 127.4(\mathrm{CH}), 126.7(\mathrm{CH}), 118.8\left(\mathrm{CH}_{2}\right), 87.4(\mathrm{CH}), 83.1(\mathrm{CH}), 82.0(\mathrm{CH}), 81.2$ $(\mathrm{CH}), 76.9(\mathrm{CH}), 75.1\left(\mathrm{CH}_{2}\right), 73.2(\mathrm{CH}), 68.8(\mathrm{CH}), 68.4\left(\mathrm{CH}_{2}\right), 37.6\left(\mathrm{CH}_{2}\right), 34.7\left(\mathrm{CH}_{2}\right)$; LRMS (ESI) $m / z 381\left([\mathrm{M}+\mathrm{Na}]^{+}, 100 \%\right)$; HRMS (ESI) calcd for $\mathrm{C}_{21} \mathrm{H}_{27} \mathrm{O}_{5}[\mathrm{M}+\mathrm{H}]^{+}:$359.1858, found: 359.1865 .

16b: $\mathrm{R}_{f}=0.53$ (ether); $[\alpha]_{\mathrm{D}}{ }^{20}-17\left(c 0.5\right.$ in $\left.\mathrm{CHCl}_{3}\right) ; v_{\max }\left(\mathrm{CHCl}_{3}\right) 3580,2892,1602,992,907,838$ $\mathrm{cm}^{-1}$; ${ }^{1} \mathrm{H}$ NMR $\left(400 \mathrm{MHz}, \mathrm{CDCl}_{3}\right) \delta 7.49-7.30$ (5H, m, Ph), 5.92-5.82 (2H, m, H-13, H-2) 5.83- 
$5.74(1 \mathrm{H}, J=11.1,8.0,3.0,2.7, \mathrm{H}-3), 5.52(1 \mathrm{H}, \mathrm{ddd}, J=17.4,1.8,1.8 \mathrm{~Hz}, \mathrm{H}-14), 5.37$ (1H, ddd, $J$ $=10.9,1.8,1.8 \mathrm{~Hz}, \mathrm{H}-14), 4.94(1 \mathrm{H}, \mathrm{d}, J=11.6 \mathrm{~Hz}, \mathrm{CH} H \mathrm{Ph}), 4.86(1 \mathrm{H}, \mathrm{d}, J=11.6 \mathrm{~Hz}, \mathrm{CHHPh})$, $4.32(1 \mathrm{H}, \mathrm{dd}, J=15.6,5.8 \mathrm{~Hz}, \mathrm{H}-1), 4.13-4.09$ (1H, m, H-12), $4.04(1 \mathrm{H}, \mathrm{ddd}, J=15.6,5.3,2.7 \mathrm{~Hz}$, $\mathrm{H}-1), 3.97$ (1H, br, H-11), $3.62(1 \mathrm{H}, \mathrm{dd}, J=9.2,8.3 \mathrm{~Hz}, \mathrm{H}-7), 3.54(1 \mathrm{H}, \mathrm{ddd}, J=11.5,9.6,4.6 \mathrm{~Hz}$, H-9), 3.38-3.29 (2H, m, H-6, H-7) 3.26 (1H, dd, $J=9.6,9.2$ Hz, H-8) 2.66 (1H, ddd, $J=16.2,8.0$, 3.4 Hz, H-4), 2.49-2.23 (2H, m, H-4, H-10) 1.78 (1H, d, J = 4.1 Hz, OH), 1.71-1.60 (1H, m, H-10); ${ }^{13} \mathrm{C}$ NMR (100 MHz, $\left.\mathrm{CDCl}_{3}\right) \delta 139.3(\mathrm{C}), 134.4(\mathrm{CH}), 131.4(\mathrm{CH}), 128.2(\mathrm{CH}), 127.9(\mathrm{CH}), 127.5$ $(\mathrm{CH}), 126.8(\mathrm{CH}), 117.6\left(\mathrm{CH}_{2}\right), 87.5(\mathrm{CH}), 82.0(\mathrm{CH}), 79.4(\mathrm{CH}), 77.4(\mathrm{CH}), 76.9(\mathrm{CH}), 75.3$ $\left(\mathrm{CH}_{2}\right), 70.2(\mathrm{CH}), 68.4\left(\mathrm{CH}_{2}\right), 67.9(\mathrm{CH}), 35.9\left(\mathrm{CH}_{2}\right), 34.7\left(\mathrm{CH}_{2}\right)$; LRMS $\left(\mathrm{CI}, \mathrm{NH}_{3}\right) \mathrm{m} / z 358\left([\mathrm{M}]^{+}\right.$, 29\%), 267 (6), 91 (100); HRMS (CI, $\mathrm{NH}_{3}$ ) calcd for $\mathrm{C}_{21} \mathrm{H}_{26} \mathrm{O}_{5}[\mathrm{M}]^{+}: 358.1780$, found: 358.1767.

\section{Acetate 17.}

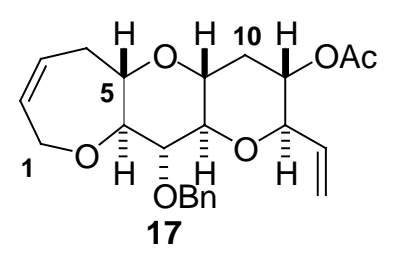

The alcohol 16a $(61 \mathrm{mg}, 0.17 \mathrm{mmol})$ was dissolved in anhydrous $\mathrm{CH}_{2} \mathrm{Cl}_{2}(2 \mathrm{~mL})$ under argon and $\mathrm{Et}_{3} \mathrm{~N}(50 \mu \mathrm{l}, 0.36 \mathrm{mmol})$ was added at rt. Acetic anhydride $(20 \mu \mathrm{l}, 0.21 \mathrm{mmol})$ was then added dropwise followed by a sub-stoichiometric amount of DMAP (1 mg). The reaction was stirred overnight before being transferred to a separating funnel containing $\mathrm{CH}_{2} \mathrm{Cl}_{2}(5 \mathrm{~mL})$ and water (5 $\mathrm{mL})$. The aqueous layer was extracted with $\mathrm{CH}_{2} \mathrm{Cl}_{2}(2 \times 5 \mathrm{~mL})$ and the combined organics were then washed with brine, dried $\left(\mathrm{MgSO}_{4}\right)$, filtered and concentrated in vacuo. The product was purified by flash column chromatography on silica gel (ether-petrol, 3:7) to give $\mathbf{1 7}$ as a colourless solid (52.6 $\mathrm{mg}, 77 \%$ ). Recrystallisation from $\mathrm{CH}_{2} \mathrm{Cl}_{2}$-hexane gave crystals suitable for X-ray crystallographic analysis: $\mathrm{R}_{f}=0.28$ (ether-hexane, 3:7); $\mathrm{Mp} 126-128^{\circ} \mathrm{C} ;[\alpha]_{\mathrm{D}}{ }^{26}+7.0\left(c 1.0, \mathrm{CHCl}_{3}\right) ; v_{\max }\left(\mathrm{CHCl}_{3}\right)$ 3697, 2877, 1737, $1602 \mathrm{~cm}^{-1}$; ${ }^{1} \mathrm{H}$ NMR (400 MHz, $\mathrm{CDCl}_{3}$ ) $\delta 7.47-7.23$ (5H, m, Ph), 5.92-5.74 (3H, m, H-2, H-3, H-13), 5.38 (1H, ddd, $J=17.3,1.4,1.2 \mathrm{~Hz}, \mathrm{H}-14), 5.25$ (1H, ddd, $J=10.9,1.2$, $1.2 \mathrm{~Hz}, \mathrm{H}-14), 4.89$ (1H, d, $J=11.6 \mathrm{~Hz}, \mathrm{CH} H \mathrm{Ph}), 4.82(1 \mathrm{H}, \mathrm{d}, J=11.6 \mathrm{~Hz}, \mathrm{CHHPh}), 4.67(1 \mathrm{H}$, ddd, $J=11.2,9.7,4.8 \mathrm{~Hz}, \mathrm{H}-11), 4.31(1 \mathrm{H}, \mathrm{dd}, J=15.6,5.9 \mathrm{~Hz}, \mathrm{H}-1), 4.04$ (1H, ddd, $J=15.6,5.1$, $2.5 \mathrm{~Hz}, \mathrm{H}-1), 3.81$ (1H, dd, $J=9.7,6.0 \mathrm{~Hz}, \mathrm{H}-12), 3.56-3.47$ (1H, m, H-7), 3.39-3.14 (4H, m, H-5, H-6, H-8, H-9), 2.65 (1H ddd, $J=16.2,7.8,3.6$ Hz, H-4), 2.51-2.43 (1H, m, H-10), 2.39-2.28 (1H, m, H-4), 2.05 (3H, s, OAc), 1.61-1.45 (1H, m, H-10); $\left.{ }^{13} \mathrm{C} \mathrm{NMR} \mathrm{(100} \mathrm{MHz,} \mathrm{CDCl}_{3}\right) \delta 169.8(\mathrm{C})$, $139.1(\mathrm{C}), 134.5(\mathrm{CH}), 131.4(\mathrm{CH}), 128.2(\mathrm{CH}), 127.9(\mathrm{CH}), 127.4(\mathrm{CH}), 126.6(\mathrm{CH}), 118.0\left(\mathrm{CH}_{2}\right)$, $87.3(\mathrm{CH}), 81.9(\mathrm{CH}), 81.3(\mathrm{CH}), 79.9(\mathrm{CH}), 76.9(\mathrm{CH}), 75.2\left(\mathrm{CH}_{2}\right), 72.7(\mathrm{CH}), 69.9(\mathrm{CH}), 68.4$ 
$\left(\mathrm{CH}_{2}\right), 35.2\left(\mathrm{CH}_{2}\right), 34.6\left(\mathrm{CH}_{2}\right) ; 21.1\left(\mathrm{CH}_{3}\right)$; HRMS (ESI) calcd for $\mathrm{C}_{23} \mathrm{H}_{29} \mathrm{O}_{6}[\mathrm{M}+\mathrm{H}]^{+}: 401.1964$, found: 401.1947.

\section{Enone 18.}

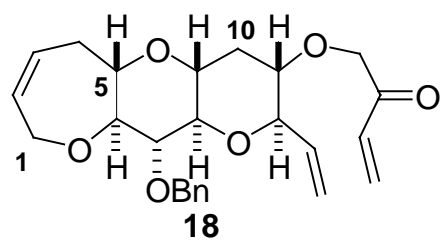

The alcohol $17(615 \mathrm{mg}, 1.72 \mathrm{mmol})$ was dissolved in anhydrous THF (30 mL). Sodium hydride (138 $\mathrm{mg}$ of $60 \%$ dispersion in mineral oil, $3.45 \mathrm{mmol}$ ) was then added followed by 1chloro-phosphoranylidene-2-propanone $(670 \mathrm{mg}, 1.90 \mathrm{mmol})$ and TBAI $(9 \mathrm{mg})$. The mixture was heated at reflux for $6 \mathrm{~h}$ and the reaction was then quenched carefully with water $(10 \mathrm{~mL})$. The reaction mixture was extracted with ether $(2 \times 20 \mathrm{~mL})$ and the combined organic layers were then washed with brine $(20 \mathrm{~mL})$, dried $\left(\mathrm{MgSO}_{4}\right)$, filtered and concentrated in vacuo to give the crude ylide as a brown oil, was used without further purification.

The phosphonium ylide was dissolved in ether $(30 \mathrm{~mL})$ and water $(10 \mathrm{~mL})$ and formaldehyde ( $387 \mu \mathrm{l}, 5.19 \mathrm{mmol}$ ) was added. The reaction was stirred for $1 \mathrm{~h}$ at $\mathrm{rt}$ and then poured into water (50 $\mathrm{mL})$. The layers were separated and the water layer was extracted ether $(2 \times 20 \mathrm{~mL})$. The combined organic layers were then washed with brine $(20 \mathrm{~mL})$, dried over $\left(\mathrm{MgSO}_{4}\right)$ and concentrated in vacuo. The product was purified by flash column chromatography on silica gel (ether-petrol, 3:7) to afford the diene 18 as a colourless solid (410 mg, 56\%, over two steps): $\mathrm{R}_{f}=0.38$ (ether-petrol, 1:1). Mp 69-72 ${ }^{\circ} \mathrm{C} ;[\alpha]_{\mathrm{D}}{ }^{26}+19\left(\right.$ c 1.0, $\left.\mathrm{CHCl}_{3}\right) ; v_{\max }\left(\mathrm{CHCl}_{3}\right) 2927,1698,990 \mathrm{~cm}^{-1} ;{ }^{1} \mathrm{H}$ NMR (400 $\left.\mathrm{MHz}, \mathrm{CDCl}_{3}\right) \delta 7.44-7.40(2 \mathrm{H}, \mathrm{m}, P h), 7.36-7.24(3 \mathrm{H}, \mathrm{m}, P h), 6.56(1 \mathrm{H}, \mathrm{dd}, J=17.6,10.7 \mathrm{~Hz}$, $\left.\mathrm{COCH}=\mathrm{CH}_{2}\right), 6.35(1 \mathrm{H}, \mathrm{dd}, J=17.6,1.4 \mathrm{~Hz}, \mathrm{COCH}=\mathrm{CHH}), 6.05(1 \mathrm{H}, \mathrm{ddd}, J=17.3,10.6,5.3 \mathrm{~Hz}$, $\left.\mathrm{CHCH}=\mathrm{CH}_{2}\right), 5.91-5.74(2 \mathrm{H}, \mathrm{m}, \mathrm{H}-2, \mathrm{H}-3), 5.84(1 \mathrm{H}, \mathrm{dd}, J=10.7,1.4 \mathrm{~Hz}, \mathrm{COCH}=\mathrm{CHH}), 5.46$ $(1 \mathrm{H}, \mathrm{ddd}, J=17.3,1.7,1.7 \mathrm{~Hz}, \mathrm{H}-14), 5.28(1 \mathrm{H}, \mathrm{ddd}, J=10.6,1.7,1.7 \mathrm{~Hz}, \mathrm{H}-14), 4.91(1 \mathrm{H}, \mathrm{d}, J=$ $11.6 \mathrm{~Hz}, \mathrm{CHHPh}), 4.82(1 \mathrm{H}, \mathrm{d}, J=11.6 \mathrm{~Hz}, \mathrm{CH} H \mathrm{Ph}), 4.37-4.25(1 \mathrm{H}, \mathrm{m}, \mathrm{H}-1) 4.34(1 \mathrm{H}, \mathrm{d}, J=16.7$ $\mathrm{Hz}, \mathrm{COCHH}), 4.28(1 \mathrm{H}, \mathrm{d}, J=16.7 \mathrm{~Hz}, \mathrm{COCH} H), 4.04(1 \mathrm{H}, \mathrm{ddd}, J=15.75 .3,2.6 \mathrm{~Hz}, \mathrm{H}-1), 3.79$ (1H, dddd, $J=7.9,5.3,1.7,1.7 \mathrm{~Hz}, \mathrm{H}-12), 3.51(1 \mathrm{H}, \mathrm{dd}, J=8.9,8.2 \mathrm{~Hz}, \mathrm{H}-7), 3.36(1 \mathrm{H}, \mathrm{dd}, J=$ 9.4, 8.2 Hz, H-6), 3.31 (1H, ddd, $J=9.4,9.2,4.0 \mathrm{~Hz}, \mathrm{H}-5), 3.24-3.18$ (1H, m, H-11), 3.23 (1H, dd, $J=9.3,8.9 \mathrm{~Hz}, \mathrm{H}-8), 3.14(1 \mathrm{H}, \mathrm{ddd}, J=11.4,9.3,4.4 \mathrm{~Hz}, \mathrm{H}-9), 2.65(1 \mathrm{H}, \mathrm{ddd}, J=16.3,7.9,4.0$ Hz, H-4), 2.56 (1H, ddd, $J 11.4$ 4.4, 4.4 Hz, H-10), 2.38-2.29 (1H, m, H-4), 1.56 (1H, ddd, $J=$ 11.4, 11.4, 11.4 Hz, H-10); ${ }^{13} \mathrm{C}$ NMR (100 MHz, $\left.\mathrm{CDCl}_{3}\right) \delta 196.8(\mathrm{C}), 139.1$ (C) $135.3(\mathrm{CH}), 132.2$ $(\mathrm{CH}), 131.5(\mathrm{CH}), 129.5\left(\mathrm{CH}_{2}\right), 128.2(\mathrm{CH}), 127.9(\mathrm{CH}), 127.5(\mathrm{CH}), 126.7(\mathrm{CH}), 117.2\left(\mathrm{CH}_{2}\right)$, 
$87.3(\mathrm{CH}), 82.0(\mathrm{CH}), 81.2(\mathrm{CH}), 80.3(\mathrm{CH}), 78.1\left(\mathrm{CH}_{2}\right), 76.9\left(\mathrm{CH}_{2}\right), 75.2(\mathrm{CH}), 73.7(\mathrm{CH}), 73.1$ (CH), $68.5\left(\mathrm{CH}_{2}\right), 35.4\left(\mathrm{CH}_{2}\right), 34.7\left(\mathrm{CH}_{2}\right)$; LRMS (ESI) $\mathrm{m} / \mathrm{z} 449\left([\mathrm{M}+\mathrm{Na}]^{+}, 100\right)$; HRMS (ESI) calcd for $\mathrm{C}_{25} \mathrm{H}_{30} \mathrm{O}_{6} \mathrm{Na}[\mathrm{M}+\mathrm{Na}]^{+}$: 449.1940, found: 449.1912. Found C, 70.30; H, 7.28; $\mathrm{C}_{25} \mathrm{H}_{30} \mathrm{O}_{6}$ requires $\mathrm{C}, 70.40 ; \mathrm{H}, 7.09$.

\section{A-D Fragment 19.}

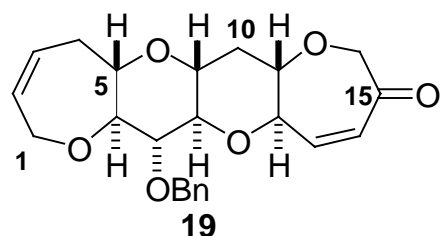

The enone 18 (229 mg, $0.537 \mathrm{mmol})$ was dissolved in anhydrous $\mathrm{CH}_{2} \mathrm{Cl}_{2}(80 \mathrm{~mL})$ under an atmosphere of argon and the ruthenium complex 9 was added $(23.0 \mathrm{mg}, 0.0271 \mathrm{mmol})$. The reaction was heated to reflux and stirred at this temperature overnight. The solvent was then removed in vасио and the residual material was purified by flash column chromatography on silica gel (ether-petrol, 3:7) giving tetracyclic ketone 19 as a colourless solid (150 mg, 70\%): Mp 132$134{ }^{\circ} \mathrm{C} ; \mathrm{R}_{f}=0.46$ (ether-petrol, 1:1); $[\alpha]_{\mathrm{D}}{ }^{26}-52$ (c 1.0, $\left.\mathrm{CHCl}_{3}\right) ; v_{\max }\left(\mathrm{CHCl}_{3}\right)$ 2878, 1667, 1608, 1006, 978, $865 \mathrm{~cm}^{-1} ;{ }^{1} \mathrm{H}$ NMR (400 MHz, $\left.\mathrm{CDCl}_{3}\right) \delta 7.37-7.18(5 \mathrm{H}, \mathrm{m}, P h), 6.48(1 \mathrm{H}, \mathrm{dd}, J=12.9$, $2.2 \mathrm{~Hz}, \mathrm{H}-13), 5.97$ (1H, dd, $J=12.9,2.6 \mathrm{~Hz}, \mathrm{H}-14), 5.84-5.76(1 \mathrm{H}, \mathrm{m}, \mathrm{H}-2), 5.74-5.66$ (1H, m, H3), 4.78 (2H, s, CHHPh), 4.32 (1H, d, $J=18.4 \mathrm{~Hz}, \mathrm{H}-16), 4.25$ (1H, dd, $J=15.6,6.1 \mathrm{~Hz}, \mathrm{H}-1), 4.19$ (1H, d, $J=18.4$ Hz, H-16), 3.99-3.91 (2H, m, H-1, H-12), 3.45-3.37 (2H, m, H-7, H-11), 3.30 (1H, dd, $J=9.1,8.2 \mathrm{~Hz}, \mathrm{H}-6), 3.23$ (1H, ddd, $J=9.6,9.2,3.9 \mathrm{~Hz}, \mathrm{H}-5), 3.16(1 \mathrm{H}, \mathrm{dd}, J=9.4,8.8 \mathrm{~Hz}, \mathrm{H}-$ 8) 3.14-3.06 (1H, m, H-9), 2.65 (1H, ddd $J=16.2,8.0,3.9$ Hz, H-4), 2.47 (1H, ddd, $J=11.5,4.3$, $4.3 \mathrm{~Hz}, \mathrm{H}-10), 2.37-2.30(1 \mathrm{H}, \mathrm{m}, \mathrm{H}-4), 1.66(1 \mathrm{H}, \mathrm{ddd}, J=11.5,11.5,11.5 \mathrm{~Hz}, \mathrm{H}-10) ;{ }^{13} \mathrm{C}$ NMR $\left(100 \mathrm{MHz}, \mathrm{CDCl}_{3}\right) \delta 201.2(\mathrm{C}), 145.0(\mathrm{CH}), 139.1(\mathrm{C}) 131.4(\mathrm{CH}), 128.3(\mathrm{CH}), 128.1(\mathrm{CH}), 127.7$ $(\mathrm{CH}), 127.5(\mathrm{CH}), 126.6(\mathrm{CH}), 87.4(\mathrm{CH}), 82.1(\mathrm{CH}), 81.6(\mathrm{CH}), 80.3(\mathrm{CH}), 78.2\left(\mathrm{CH}_{2}\right), 77.1(\mathrm{CH})$, $77.0\left(\mathrm{CH}_{2}\right), 75.4(\mathrm{CH}), 73.0(\mathrm{CH}), 68.4\left(\mathrm{CH}_{2}\right), 36.3\left(\mathrm{CH}_{2}\right), 34.6\left(\mathrm{CH}_{2}\right)$; LRMS $\left(\mathrm{CI}, \mathrm{NH}_{3}\right) \mathrm{m} / \mathrm{z} 398$ ([M] $\left.]^{+}, 12 \%\right), 214$ (100); HRMS (CI, $\mathrm{NH}_{3}$ ) calcd for $\mathrm{C}_{23} \mathrm{H}_{26} \mathrm{O}_{6}[\mathrm{M}]^{+}$: 398.1729, found: 398.1716. Found $\mathrm{C}, 69.11 ; \mathrm{H}, 6.72 ; \mathrm{C}_{23} \mathrm{H}_{26} \mathrm{O}_{6}$ requires $\mathrm{C}, 69.33 ; \mathrm{H}, 6.58$. 


\section{Ketones 20a and 20b.}

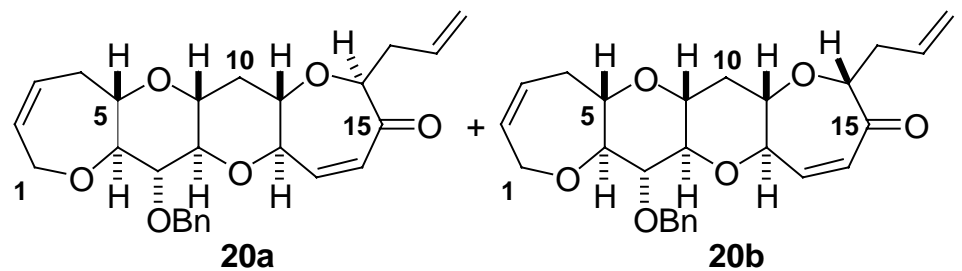

The enone 19 (184 mg, $0.462 \mathrm{mmol})$ was dissolved in anhydrous benzene $(8 \mathrm{~mL}) . N, N$ Dimethylhydrazine $(179 \mu \mathrm{l}, 2.36 \mathrm{mmol})$ and $\mathrm{MgSO}_{4}(300 \mathrm{mg}, 2.49 \mathrm{mmol}$ ) were followed by dropwise addition of glacial acetic acid $(266 \mu \mathrm{l}, 4.65 \mathrm{mmol})$ at rt. After 20 min additional $\mathrm{MgSO}_{4}$ $(150 \mathrm{mg}, 1.25 \mathrm{mmol})$ was added and the resulting mixture was stirred for a further $30 \mathrm{~min}$. The reaction was quenched with a sat. aqueous solution of $\mathrm{NaHCO}_{3}(5 \mathrm{~mL})$ and extracted with EtOAc $(3 \times 5 \mathrm{~mL})$. The combined organic layers were washed in brine $(15 \mathrm{~mL})$ and then dried $\left(\mathrm{MgSO}_{4}\right)$, filtered and concentrated in vacuo. Purification of the residual material by flash column chromatography on $10 \%$ deactivated alumina (petrol-ether, 1:1) gave the hydrazone as a yellow solid.

The crude hydrazone was dissolved in anhydrous THF $(8 \mathrm{~mL})$ under argon and the solution was cooled to $-78{ }^{\circ} \mathrm{C}$. $t$-BuLi ( $408 \mu \mathrm{l}$ of a $1.5 \mathrm{M}$ in pentane) was added dropwise and the reaction was allowed to stir for $5 \mathrm{~min}$ at $-78{ }^{\circ} \mathrm{C}$. Allyl bromide $(200 \mu \mathrm{l}, 2.31 \mathrm{mmol})$ was added and the mixture was stirred for a further $20 \mathrm{~min}$. The reaction was then quenched by the addition of water $(5 \mathrm{~mL})$. The mixture was transferred to a separating funnel and the aqueous layer was separated and extracted with ether $(2 \times 5 \mathrm{~mL})$. The combined organic layers were washed with brine $(15 \mathrm{~mL})$, dried over $\left(\mathrm{MgSO}_{4}\right)$, filtered and concentrated in vacuo. The product was used without further purification.

The crude product was dissolved in THF $(300 \mu \mathrm{l})$ and water $(150 \mu \mathrm{l})$. Solid $\mathrm{CuCl}_{2}(223 \mathrm{mg}, 1.66$ mmol) was added and the reaction was stirred for $1 \mathrm{~h}$ at $\mathrm{rt}$. The mixture was then transferred to a separating funnel and diluted with water $(2 \mathrm{~mL})$ and ether $(2 \mathrm{~mL})$. The combined organic layers were washed with brine, dried $\left(\mathrm{MgSO}_{4}\right)$, filtered and concentrated in vacuo. The product was purified by flash column chromatography on silica gel (ether-petrol, 3:7) giving an inseparable mixture of the enones $20 \mathbf{a}$ and $20 \mathbf{b}$ ( $4: 1$ ratio) as a colourless solid ( $89.4 \mathrm{mg}$, $44 \%$, over 3 steps).

A mixture of enones $20 \mathbf{a}$ and $20 \mathbf{b}(63.5 \mathrm{mg}, 0.144 \mathrm{mmol})$, was dissolved in $\mathrm{C}_{6} \mathrm{D}_{6}(7.5 \mathrm{~mL})$ and 2-t-butyl-1,1,3,3-tetramethylguanidine (Barton's base) $(35.3 \mu \mathrm{l}, 0.288 \mathrm{mmol})$ was added and the mixture was heated to $60{ }^{\circ} \mathrm{C}$ for $12 \mathrm{~h}$ and the left at $\mathrm{rt}$ for a further $9 \mathrm{~h}$. The mixture was filtered through a short plug of silica gel and concentrated in vacuo. Purification by flash column chromatography (silica, 1:1 hexane/ether) gave the enones $\mathbf{2 0 a}$ and $\mathbf{2 0 b}$ (1:4 ratio) as a colourless oil (40.0 mg, 63\%). 
20a: $\mathrm{Mp} 84-86{ }^{\circ} \mathrm{C} ; \mathrm{R}_{f}=0.46$ (petrol-ether, 1:1); $[\alpha]_{\mathrm{D}}{ }^{26}-60\left(\right.$ c 1.0, $\left.\mathrm{CHCl}_{3}\right) ; v_{\max }\left(\mathrm{CHCl}_{3}\right) 3695$, 2928, 2875, 1661, 1602, 909, $866 \mathrm{~cm}^{-1}$; ${ }^{1} \mathrm{H} \mathrm{NMR}\left(400 \mathrm{MHz}, \mathrm{CDCl}_{3}\right) \delta$ 7.45-7.27 (5H, m, Ph), 6.54 $(1 \mathrm{H}, \mathrm{dd}, J=12.6,2.5 \mathrm{~Hz}, \mathrm{H}-13), 6.05$ (1H, dd, $J=12.6,2.6 \mathrm{~Hz}, \mathrm{H}-14), 5.93-5.74$ (3H, m, H-2, H3, H-18), 5.20 (1H, dddd, $J=17.1,1.5,1.5,1.5 \mathrm{~Hz}, \mathrm{H}-19), 5.14$ (1H, dd, $J=10.2,1.5 \mathrm{~Hz}, \mathrm{H}-19)$, $4.86(2 \mathrm{H}, \mathrm{s}, \mathrm{CHHPh}) 4.32(1 \mathrm{H}, \mathrm{dd}, J=10.0,3.9 \mathrm{~Hz}, \mathrm{H}-16), 4.31(1 \mathrm{H}, \mathrm{dd}, J=15.3,6.1 \mathrm{~Hz}, \mathrm{H}-1)$, 4.06-4.03 (1H, m, H-1), 4.02 (1H, ddd, J = 9.0, 2.6, 2.5 Hz, H-12), 3.63 (1H, ddd, $J=11.3,9.0$, 4.6 Hz, H-11), 3.49 (1H, dd, $J=8.7,8.2 \mathrm{~Hz}, \mathrm{H}-7), 3.37$ (1H, dd, $J=9.1,8.2 \mathrm{~Hz}, \mathrm{H}-6), 3.31$ (1H, ddd, $J$ = 9.6, 9.1, 3.9 Hz, H-5), $3.22(1 \mathrm{H}, \mathrm{dd}, J=9.3,8.7 \mathrm{~Hz}, \mathrm{H}-8), 3.22-3.14$ (1H, m, H-9), 2.78-2.70 (1H, m, H-17), 2.66 (1H, ddd, $J=16.2,7.9,3.9$ Hz, H-4), 2.56-2.47 (1H, m, H-17), 2.42 (1H, ddd, $J=11.4,4.4,4.2 \mathrm{~Hz}, \mathrm{H}-10), 2.37-2.30$ (1H, m, H-4), 1.65 (1H, ddd $J=11.4,11.4,11.4 \mathrm{~Hz}, \mathrm{H}-10)$; ${ }^{13} \mathrm{C}$ NMR (100 MHz, $\left.\mathrm{CDCl}_{3}\right) \delta 201.8(\mathrm{C}), 144.2(\mathrm{CH}) 139.1(\mathrm{C}), 134.1(\mathrm{CH}), 131.4(\mathrm{CH}), 128.5$ $(\mathrm{CH}), 128.3(\mathrm{CH}), 127.7(\mathrm{CH}), 127.5(\mathrm{CH}), 126.7(\mathrm{CH}), 118.0\left(\mathrm{CH}_{2}\right), 87.7(\mathrm{CH}), 83.4(\mathrm{CH}), 82.1$ $(\mathrm{CH}), 81.5(\mathrm{CH}), 79.7(\mathrm{CH}), 76.9(\mathrm{CH}), 75.3\left(\mathrm{CH}_{2}\right), 73.1(\mathrm{CH}), 72.0(\mathrm{CH}), 68.4\left(\mathrm{CH}_{2}\right), 36.2\left(\mathrm{CH}_{2}\right)$, $35.3\left(\mathrm{CH}_{2}\right)$, $34.6\left(\mathrm{CH}_{2}\right)$; HRMS (ESI) calcd for $\mathrm{C}_{26} \mathrm{H}_{31} \mathrm{O}_{6}[\mathrm{M}+\mathrm{H}]^{+}$: 439.2121, found: 439.2151.

20b: $\mathrm{R}_{f}=0.46$ (petrol-ether, 1:1); $[\alpha]_{\mathrm{D}}{ }^{26}-6\left(c 0.5, \mathrm{CHCl}_{3}\right) ; v_{\max }\left(\mathrm{CHCl}_{3}\right) 2929,2873,1721,1665$, $1096 \mathrm{~cm}^{-1} ;{ }^{1} \mathrm{H}$ NMR $\left(400 \mathrm{MHz}, \mathrm{CDCl}_{3}\right) \delta 7.42-7.23(5 \mathrm{H}, \mathrm{m}, P h), 6.49(1 \mathrm{H}, \mathrm{dd}, J=12.8,2.5 \mathrm{~Hz}$, H-13), 6.00 (1H, dd, $J=12.8,2.6 \mathrm{~Hz}, \mathrm{H}-14), 5.91-5.75$ (3H, m, H-2, H-3, H-18), 5.10 (1H, ddd, $J=$ 17.7, 1.2, 0.5 Hz, H-19), 5.06 (1H, ddd, $J=10.2,1.2,1.0 \mathrm{~Hz}, \mathrm{H}-19), 4.85$ (2H, s, CHHPh), 4.30 (1H, dd, $J=15.5,5.9 \mathrm{~Hz}, \mathrm{H}-1), 4.24$ (1H, dd $J=7.6,4.2 \mathrm{~Hz}, \mathrm{H}-16), 4.06-4.00$ (1H, m, H-1), 3.96 (1H, ddd, $J=8.9,2.6,2.5 \mathrm{~Hz}, \mathrm{H}-12), 3.49$ (1H, dd, $J=9.0,9.0 \mathrm{~Hz}, \mathrm{H}-7), 3.46$ (1H, ddd, $J=11.4$, 8.9, 4.6 Hz, H-11), 3.37 (1H, dd, $J=9.4,9.0 \mathrm{~Hz}, \mathrm{H}-8), 3.30$ (1H, ddd, $J=9.8,9.4,4.0 \mathrm{~Hz}, \mathrm{H}-5)$, $3.23(1 \mathrm{H}, \mathrm{dd}, J=9.4,9.0 \mathrm{~Hz}, \mathrm{H}-6), 3.15$ (1H, ddd, $J=11.6,9.4,4.4 \mathrm{~Hz}, \mathrm{H}-9), 2.65$ (1H, ddd, $J=$ 16.2, 7.8, 4.0 Hz, H-4), 2.61-2.54 (1H, m, H-17), 2.48-2.39 (2H, m, H-10, H-17), 2.39-2.30 (1H, m, H-4), $1.68(1 \mathrm{H}$, ddd $J=11.6,11.6,11.4 \mathrm{~Hz}, \mathrm{H}-10) ;{ }^{13} \mathrm{C} \mathrm{NMR}\left(100 \mathrm{MHz}, \mathrm{CDCl}_{3}\right) \delta 203.1(\mathrm{C})$, $143.6(\mathrm{CH}) 139.1(\mathrm{C}), 133.3(\mathrm{CH}), 131.4(\mathrm{CH}), 128.5(\mathrm{CH}), 128.3(\mathrm{CH}), 128.2(\mathrm{CH}), 127.7(\mathrm{CH})$, $126.7(\mathrm{CH}), 117.8\left(\mathrm{CH}_{2}\right), 87.5(\mathrm{CH}), 86.8(\mathrm{CH}), 82.2(\mathrm{CH}), 81.6(\mathrm{CH}), 79.8(\mathrm{CH}), 77.2(\mathrm{CH}), 76.8$ $(\mathrm{CH}), 75.4\left(\mathrm{CH}_{2}\right), 73.1(\mathrm{CH}), 68.5\left(\mathrm{CH}_{2}\right), 37.7\left(\mathrm{CH}_{2}\right), 36.2\left(\mathrm{CH}_{2}\right), 34.6\left(\mathrm{CH}_{2}\right)$; HRMS (ESI) calcd for $\mathrm{C}_{26} \mathrm{H}_{31} \mathrm{O}_{6}[\mathrm{M}+\mathrm{H}]^{+}$: 439.2121, found: 439.2162 . 


\section{Alcohol 21.}

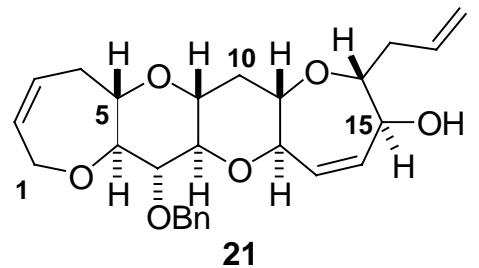

Cerium trichloride heptahydrate $(34 \mathrm{mg}, 0.091 \mathrm{mmol}$ ) was added to a solution of the enones 20a and 20b (40 mg of a 1:4 mixture, $0.091 \mathrm{mmol})$ in anhydrous $\mathrm{MeOH}(5 \mathrm{~mL})$ under argon at $-78{ }^{\circ} \mathrm{C}$. Sodium borohydride $(4.0 \mathrm{mg}, 0.11 \mathrm{mmol}$ ) was added and the reaction was allowed to stir for $1 \mathrm{~h}$ at $-78{ }^{\circ} \mathrm{C}$. The solution was then transferred to a separating funnel containing water $(1 \mathrm{~mL})$ and ether $(3 \mathrm{~mL})$ and the aqueous layer was extracted with ether $(2 \times 3 \mathrm{~mL})$. The combined organic layers were washed with brine $(5 \mathrm{~mL})$, dried $\left(\mathrm{MgSO}_{4}\right)$ and concentrated in vacuo. Purification of the residue by flash column chromatography on silica gel (petrol-ether, 6:4) gave the alcohol 21 and an isomeric alcohol derived from ketone 20a as a white solid $(37.7 \mathrm{mg}, 94 \%)$. The alcohols were separable by chromatography and recrystallisation of the alcohol 21 from $\mathrm{CH}_{2} \mathrm{Cl}_{2}$-hexane gave crystals suitable for X-ray crystallographic analysis: $\mathrm{Mp} 114-116{ }^{\circ} \mathrm{C} ; \mathrm{R}_{f}=0.30$ (petrol-ether, 1:1); $[\alpha]_{\mathrm{D}}{ }^{20}-21\left(c 1.0, \mathrm{CHCl}_{3}\right) ; v_{\max }\left(\mathrm{CHCl}_{3}\right) 3707,3624,2928,1643,873 \mathrm{~cm}^{-1} ;{ }^{1} \mathrm{H}$ NMR $(500 \mathrm{MHz}$, $\left.\mathrm{CDCl}_{3}\right) \delta$ 7.43-7.40 (2H, m, Ph), 7.36-7.32 (2H, m, Ph), 7.30-7.25 (1H, m, Ph), 5.94 (1H, dddd, $J$ $=17.2,10.1,6.9,6.9 \mathrm{~Hz}, \mathrm{H}-18), 5.90-5.84(1 \mathrm{H}, \mathrm{m}, \mathrm{H}-3), 5.81-5.75(1 \mathrm{H}, \mathrm{m}, \mathrm{H}-2), 5.69(2 \mathrm{H}, \mathrm{s}, \mathrm{H}-$ 13, H-14), 5.13 (1H, dd, $J=17.2,1.0 \mathrm{~Hz}, \mathrm{H}-19), 5.08$ (1H, dd, $J=10.1,1.0 \mathrm{~Hz}, \mathrm{H}-19), 4.88$ (1H, d, $J=11.7 \mathrm{~Hz}, \mathrm{C} H \mathrm{HPh}), 4.84(1 \mathrm{H}, \mathrm{d}, J=11.7 \mathrm{~Hz}, \mathrm{C} H \mathrm{HPh}), 4.30(1 \mathrm{H}, \mathrm{dd}, J=15.3,5.9 \mathrm{~Hz}, \mathrm{H}-1)$, 4.19-4.13 (1H, m, H-15), 4.02 (1H, ddd, $J=15.3,5.2,2.1 \mathrm{~Hz}, \mathrm{H}-1), 3.86$ (1H, dd, $J=9.1,1.8 \mathrm{~Hz}$, H-12), 3.48 (1H, dd, $J=8.9,8.5$ Hz, H-7), 3.37 (1H, ddd, $J=8.8,8.4,3.1 \mathrm{~Hz}, \mathrm{H}-16), 3.35$ (1H, dd, $J=8.5,8.4$ Hz, H-6), 3.33-3.25 (2H, m, H-5, H-11), 3.16-3.09 (2H, m, H-8, H-9), 2.64 (1H, ddd, $J$ $=16.3,8.0,4.0 \mathrm{~Hz}, \mathrm{H}-4), 2.59-2.53$ (1H, m, H-17), 2.38-2.22 (3H, m, H-4, H-10, H-17), 1.75 (1H, m, OH), $1.56(1 \mathrm{H}$, ddd, $J$ 11.6, 11.6, $11.6 \mathrm{~Hz}, \mathrm{H}-10) ;{ }^{13} \mathrm{C}$ NMR $\left(100 \mathrm{MHz}, \mathrm{CDCl}_{3}\right) \delta 139.2(\mathrm{C})$, $134.9(\mathrm{CH}), 134.2(\mathrm{CH}), 131.4(\mathrm{CH}), 131.3(\mathrm{CH}), 128.2(\mathrm{CH}), 127.8(\mathrm{CH}), 127.5(\mathrm{CH}), 126.8$ $(\mathrm{CH}), 117.1\left(\mathrm{CH}_{2}\right), 87.5(\mathrm{CH}), 84.4(\mathrm{CH}), 82.2(\mathrm{CH}), 81.0(\mathrm{CH}), 80.4(\mathrm{CH}), 78.7(\mathrm{CH}), 76.9(\mathrm{CH})$, $75.2\left(\mathrm{CH}_{2}\right), 73.7(\mathrm{CH}), 73.3(\mathrm{CH}), 68.4\left(\mathrm{CH}_{2}\right), 37.6\left(\mathrm{CH}_{2}\right), 36.7\left(\mathrm{CH}_{2}\right), 34.7\left(\mathrm{CH}_{2}\right) ; \mathrm{HRMS}(\mathrm{ESI})$ calcd for $\mathrm{C}_{26} \mathrm{H}_{32} \mathrm{O}_{6} \mathrm{Na}[\mathrm{M}+\mathrm{Na}]^{+}:$463.2091, found: 463.2100 . 


\section{Enone 22.}

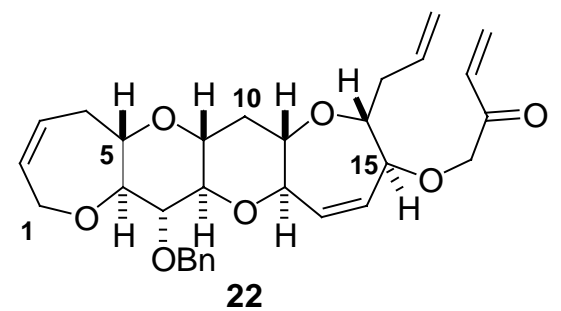

The alcohol 21 (31 mg, $0.070 \mathrm{mmol}$ ) was dissolved in THF (2 mL) and sodium hydride (3.4 mg, $0.080 \mathrm{mmol}$ ) was added followed by 1-chloro-phosphoranylidene-2-propanone (50.0 mg, 0.14 mmol) and TBAI (1 mg, $5 \mathrm{~mol} \%)$. The mixture was heated under reflux for $6 \mathrm{~h}$. The reaction was then quenched carefully with water $(1 \mathrm{~mL})$ and extracted with ether $(2 \times 2 \mathrm{~mL})$. The combined organic layers were washed with brine $(2 \mathrm{~mL})$, dried $\left(\mathrm{MgSO}_{4}\right)$, filtered and concentrated in vacuo to give the phosphorane intermediate as a brown oil which was used without further purification. $\mathbf{R}_{f}=$ $0.32\left(\mathrm{MeOH}-\mathrm{CHCl}_{3}, 1: 10\right)$.

The crude phosphorane was dissolved in ether $(2 \mathrm{~mL})$ and water $(800 \mu \mathrm{l})$ and aqueous formaldehyde ( $57 \mu \mathrm{l}$ of a $37 \% \mathrm{w} / \mathrm{v}$ aqueous solution, $0.70 \mathrm{mmol}$ ) were added. The solution was stirred for $1.5 \mathrm{~h}$ then partitioned between ether $(2 \mathrm{~mL})$ and water $(1 \mathrm{~mL})$. The aqueous layer was separated and extracted with ether $(2 \times 2 \mathrm{~mL})$. The combined organic layers were washed with brine $(2 \mathrm{~mL})$, dried $\left(\mathrm{MgSO}_{4}\right)$, filtered and concentrated in vacuo. Purification of the residue by flash column chromatography on silica gel (petrol-ether, 1:1) afforded the enone 22 as a colourless oil (19.6 mg, 55\%): $\mathrm{R}_{f}=0.28$ (petrol-ether, 1:1); $[\alpha]_{\mathrm{D}}{ }^{20}+1.4\left(c 1.0, \mathrm{CHCl}_{3}\right) ; v_{\max }\left(\mathrm{CHCl}_{3}\right) 2926,1780$, $1602 \mathrm{~cm}^{-1} ;{ }^{1} \mathrm{H}$ NMR (500 MHz, $\left.\mathrm{CDCl}_{3}\right) \delta$ 7.43-7.39 (2H, m, Ph), 7.36-7.32 (2H, m, Ph), 7.29$7.25(1 \mathrm{H}, \mathrm{m}, P h), 6.55\left(1 \mathrm{H}, \mathrm{dd}, J=17.6,10.7 \mathrm{~Hz}, \mathrm{COCH}=\mathrm{CH}_{2}\right), 6.35(1 \mathrm{H}, \mathrm{ddd}, J=17.6,1.3 \mathrm{~Hz}$, $\mathrm{COCH}=\mathrm{CH} H), 5.94-5.84(2 \mathrm{H}, \mathrm{m}, \mathrm{H}-2, \mathrm{H}-3), 5.86(1 \mathrm{H}, \mathrm{dd}, J=10.7,1.3 \mathrm{~Hz}, \mathrm{COCH}=\mathrm{CHH}), 5.82-$ 5.75 (3H, m, H-13, H-14, H-18), 5.08 (1H, dd, $J=17.5,1.7 \mathrm{~Hz}, \mathrm{H}-19), 5.05$ (1H, dd, $J=10.2,1.7$ $\mathrm{Hz}, \mathrm{H}-19), 4.88(1 \mathrm{H}, \mathrm{d}, J=11.6 \mathrm{~Hz}, \mathrm{CH} H \mathrm{Ph}), 4.84(1 \mathrm{H}, \mathrm{d}, J=11.6 \mathrm{~Hz}, \mathrm{CH} H \mathrm{Ph}), 4.40(1 \mathrm{H}, \mathrm{d}, J=$ $\left.16.7 \mathrm{~Hz}, \mathrm{CHHCOCH}=\mathrm{CH}_{2}\right), 4.29(1 \mathrm{H}, \mathrm{dd}, J=15.6,6.1 \mathrm{~Hz}, \mathrm{H}-1), 4.28(1 \mathrm{H}, \mathrm{d}, J=16.7 \mathrm{~Hz}$, $\left.\mathrm{C} H \mathrm{HCOCH}=\mathrm{CH}_{2}\right), 4.01(1 \mathrm{H}, \mathrm{ddd} J=15.6,5.3,2.7 \mathrm{~Hz}, \mathrm{H}-1), 3.89(1 \mathrm{H}, \mathrm{ddd}, J=8.8,3.6,1.9 \mathrm{~Hz}$, H-15), 3.85 (1H, ddd, $J=9.0,3.3,2.0 \mathrm{~Hz}, \mathrm{H}-12), 3.54$ (1H, ddd, $J=8.8,8.6,2.9 \mathrm{~Hz}, \mathrm{H}-16), 3.48$ $(1 \mathrm{H}, \mathrm{dd}, J=8.6,8.3 \mathrm{~Hz}, \mathrm{H}-7), 3.34$ (1H, dd, $J=9.0,8.3 \mathrm{~Hz}, \mathrm{H}-6), 3.33-3.25$ (2H, m, H-5, H-11), 3.16-3.08 (2H, m, H-8, H-9), 2.64 (1H, ddd, $J=16.3,7.8,3.9$ Hz, H-4), 2.63-2.57 (1H, m, H-17), 2.38-2.22 (3H, m, H-4, H-10, H-17), 1.54 (1H, ddd, $J=11.4,11.4,11.4 \mathrm{~Hz}, \mathrm{H}-10) ;{ }^{13} \mathrm{C}$ NMR $(125$ $\left.\mathrm{MHz}, \mathrm{CDCl}_{3}\right) \delta 196.1(\mathrm{C}), 139.2(\mathrm{CH}), 134.9(\mathrm{C}), 132.7(\mathrm{CH}), 132.4(\mathrm{CH}), 131.4(\mathrm{CH}), 131.1$ $(\mathrm{CH}), 129.4\left(\mathrm{CH}_{2}\right), 128.2(\mathrm{CH}), 127.8(\mathrm{CH}), 127.5(\mathrm{CH}), 126.8(\mathrm{CH}), 117.0\left(\mathrm{CH}_{2}\right), 87.5(\mathrm{CH}), 83.2$ $(\mathrm{CH}), 82.2(\mathrm{CH}), 81.8(\mathrm{CH}), 81.0(\mathrm{CH}), 80.4(\mathrm{CH}), 78.5(\mathrm{CH}), 76.8(\mathrm{CH}), 75.2\left(\mathrm{CH}_{2}\right), 73.7\left(\mathrm{CH}_{2}\right)$, 
$73.2(\mathrm{CH}), 68.4\left(\mathrm{CH}_{2}\right), 37.2\left(\mathrm{CH}_{2}\right), 36.7\left(\mathrm{CH}_{2}\right), 34.7\left(\mathrm{CH}_{2}\right)$; HRMS (ESI) calcd for $\mathrm{C}_{30} \mathrm{H}_{36} \mathrm{O}_{7} \mathrm{Na}$ $[\mathrm{M}+\mathrm{Na}]^{+}:$531.2359, found: 531.2335.

\section{A-E Fragment 23.}

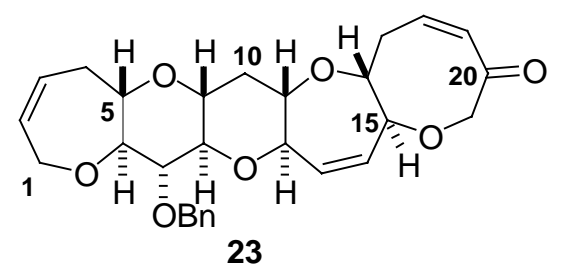

The enone 22 (7.5 mg, $(0.015 \mathrm{mmol})$ was dissolved in dry $\mathrm{CH}_{2} \mathrm{Cl}_{2}(2 \mathrm{~mL})$ and the ruthenium complex 9 was added $(1.2 \mathrm{mg}, 0.0015 \mathrm{mmol})$. The reaction mixture was heated to $40{ }^{\circ} \mathrm{C}$ for $1 \mathrm{~h}$ under an atmosphere of argon. The solvent was then removed in vacuo and the product was purified by flash column chromatography on silica gel (ether-petrol, 1:1) to give the pentacyclic ketone $\mathbf{2 3}$ as a white solid (3.5 mg, 50\%): $\mathrm{Mp} 144-146{ }^{\circ} \mathrm{C} ; \mathrm{R}_{f}=0.50$ (petrol-ether, $\left.1: 1\right) ;[\alpha]_{\mathrm{D}}{ }^{18}-0.8(c 0.5$, $\left.\mathrm{CHCl}_{3}\right) ; v_{\max }\left(\mathrm{CHCl}_{3}\right) 2927,1696,1598 \mathrm{~cm}^{-1} ;{ }^{1} \mathrm{H} \mathrm{NMR}\left(500 \mathrm{MHz}, \mathrm{CDCl}_{3}\right) \delta 7.43-7.40(2 \mathrm{H}, \mathrm{m}$, $P h), 7.37-7.32$ (2H, m, Ph), 7.30-7.26 (1H, m, Ph), $6.46(1 \mathrm{H}, \mathrm{ddd}, J=12.5,7.9,6.9 \mathrm{~Hz}, \mathrm{H}-18)$, $5.89(1 \mathrm{H}, \mathrm{dd}, J=12.5,1.0 \mathrm{~Hz}, \mathrm{H}-19), 5.90-5.84(1 \mathrm{H}, \mathrm{m}, \mathrm{H}-2), 5.81-5.75(1 \mathrm{H}, \mathrm{m}, \mathrm{H}-3), 5.78$ (2H, s, H-13, H-14), 4.87 (1H, d, $J=11.6 \mathrm{~Hz}, \mathrm{CH} H \mathrm{Ph}), 4.84(1 \mathrm{H}, \mathrm{d}, J=11.6 \mathrm{~Hz}, \mathrm{CHHPh}), 4.46$ (1H, d, $J$ $=18.1 \mathrm{~Hz}, \mathrm{H}-21), 4.30(1 \mathrm{H}, \mathrm{dd}, J=15.5,5.8 \mathrm{~Hz}, \mathrm{H}-1), 4.25(1 \mathrm{H}, \mathrm{d}, J=9.3 \mathrm{~Hz}, \mathrm{H}-15), 4.22(1 \mathrm{H}, \mathrm{d}$, $J=18.1 \mathrm{~Hz}, \mathrm{H}-21), 4.02(1 \mathrm{H}, \mathrm{ddd}, J=15.5,5.2,2.6 \mathrm{~Hz}, \mathrm{H}-1), 3.84(1 \mathrm{H}, \mathrm{d}, J=9.1 \mathrm{~Hz}, \mathrm{H}-12), 3.59$ $(1 \mathrm{H}, \mathrm{ddd}, J=9.3,9.2,1.4 \mathrm{~Hz}, \mathrm{H}-16), 3.48(1 \mathrm{H}, \mathrm{dd}, J=8.6,8.4 \mathrm{~Hz}, \mathrm{H}-7), 3.35$ (1H, dd, $J=9.1,8.4$ Hz, H-6), 3.34-3.30 (1H, m, H-11), 3.29 (1H, ddd, $J=9.6,9.1,3.8$ Hz, H-5), 3.15-3.09 (2H, m, H8, H-9), 2.83-2.75 (1H, m, H-17), 2.69-2.59 (2H, m, H-4, H-17), 2.38-2.28 (2H, m, H-4, H-10), $1.53(1 \mathrm{H}, \mathrm{ddd}, J=11.4,11.4,11.4 \mathrm{~Hz}, \mathrm{H}-10) ;{ }^{13} \mathrm{C} \mathrm{NMR}\left(125 \mathrm{MHz}, \mathrm{CDCl}_{3}\right) \delta 203.7(\mathrm{C}), 139.4$ $(\mathrm{CH}), 139.2(\mathrm{C}), 132.9(\mathrm{CH}), 132.4(\mathrm{CH}), 131.4(\mathrm{CH}), 128.7(\mathrm{CH}), 128.3(\mathrm{CH}), 127.8(\mathrm{CH}), 127.5$ $(\mathrm{CH}), 126.7(\mathrm{CH}), 87.4(\mathrm{CH}), 85.9(\mathrm{CH}), 82.1(\mathrm{CH}), 81.0(\mathrm{CH}), 80.3(\mathrm{CH}), 80.0(\mathrm{CH}), 78.6(\mathrm{CH})$, $77.3\left(\mathrm{CH}_{2}\right), 76.9(\mathrm{CH}), 75.3\left(\mathrm{CH}_{2}\right), 73.1(\mathrm{CH}), 68.4\left(\mathrm{CH}_{2}\right), 37.5\left(\mathrm{CH}_{2}\right), 36.9\left(\mathrm{CH}_{2}\right), 34.6\left(\mathrm{CH}_{2}\right)$; HRMS (ESI) calcd for $\mathrm{C}_{28} \mathrm{H}_{32} \mathrm{O}_{7} \mathrm{Na}[\mathrm{M}+\mathrm{Na}]^{+}:$503.2046, found: 503.2029. 

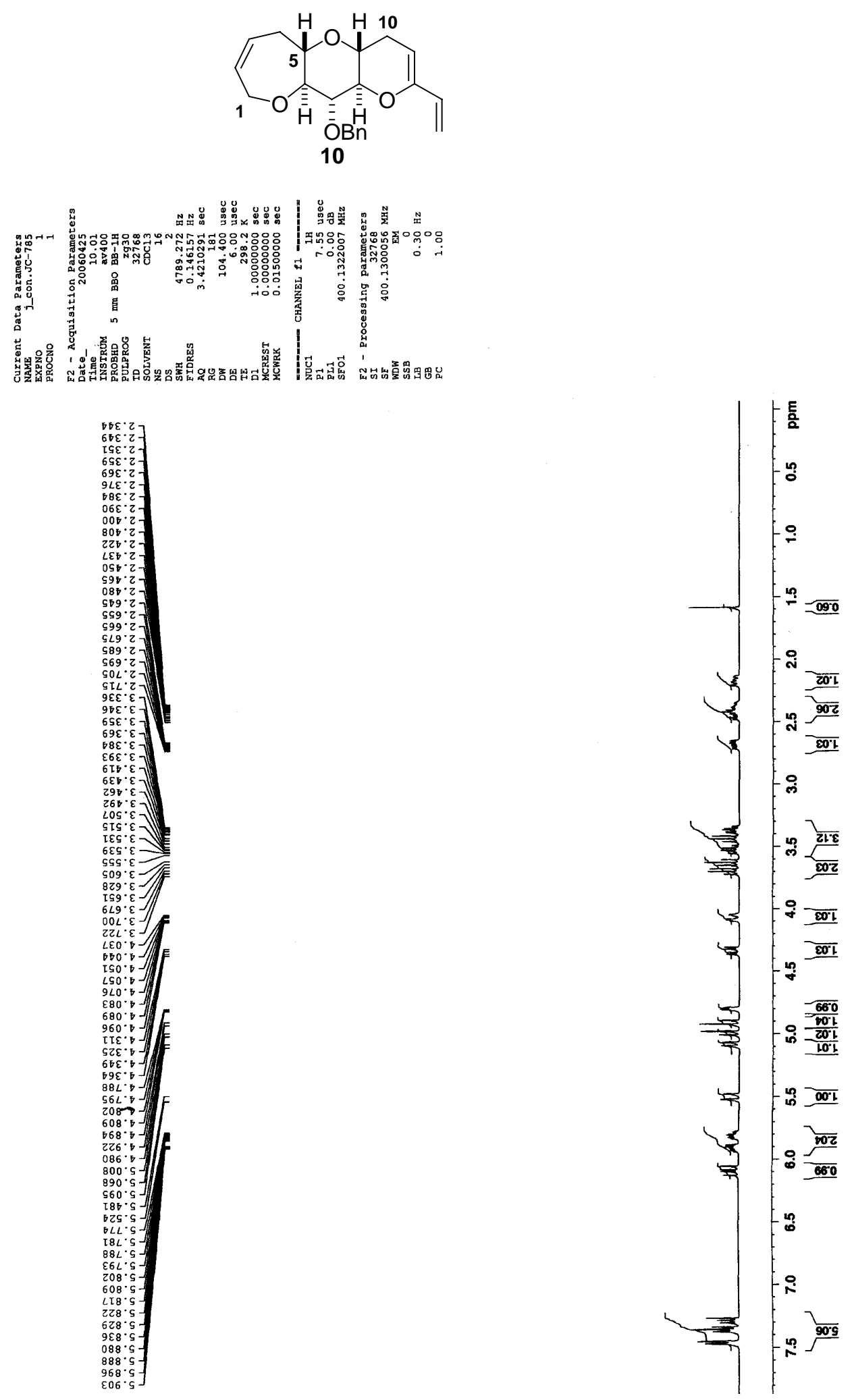


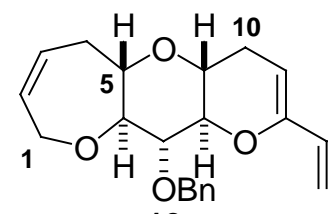

10

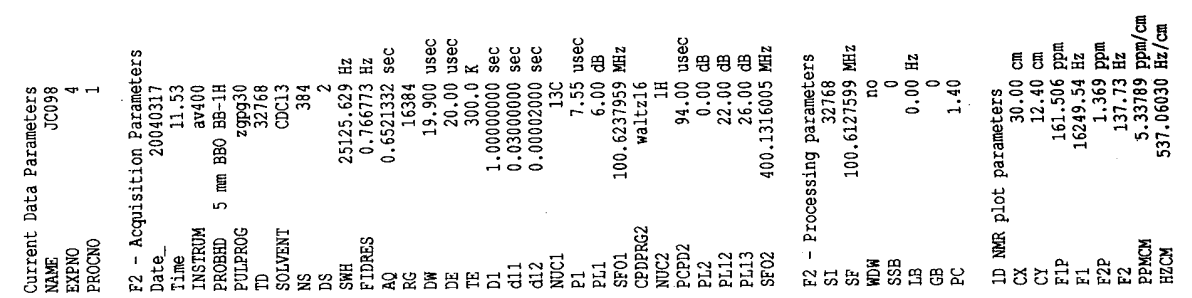

$966 \cdot L 2-$

$689^{\circ} 6 \varepsilon-$

098.89--

SOS $\pi L>$

$\angle 6 \varepsilon: 9 L>$

201.92

$\theta 8+t L]$

$29 L: 8 L]$

BLT. $\angle 8$

โ8\&:00T—-

$\varepsilon \varepsilon Z^{\circ} \varepsilon \tau I-$

$256^{\circ} 927-$

$\angle 65^{\circ} \angle 2 \mathrm{~T}$

29E. $8 \mathrm{ZT}$

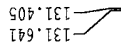

6ฐI. $6 \varepsilon I-$

$229 \cdot$ OST

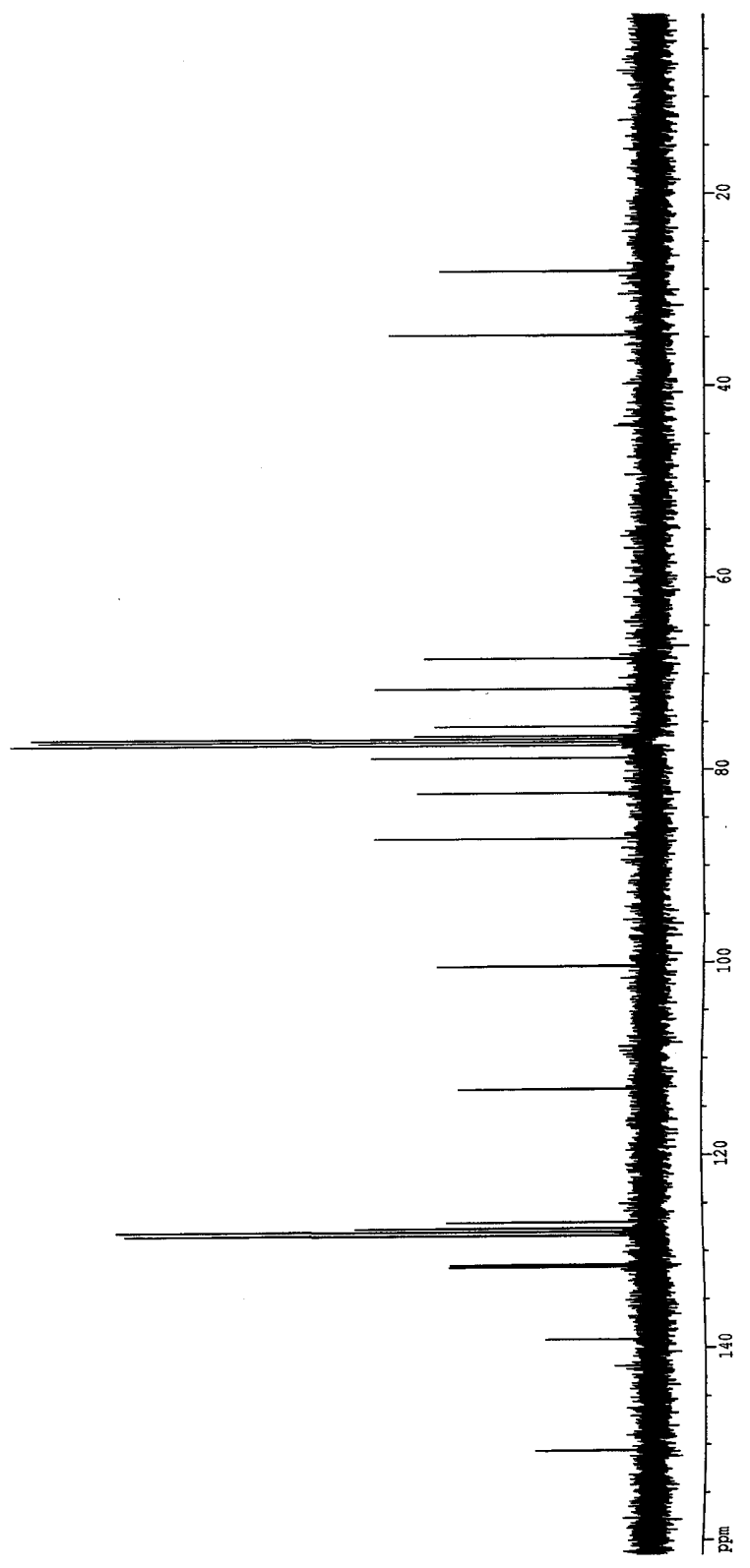



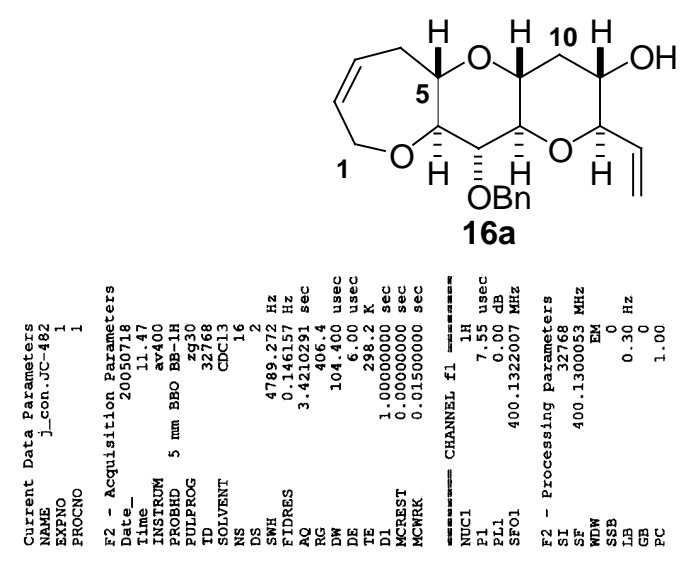

;

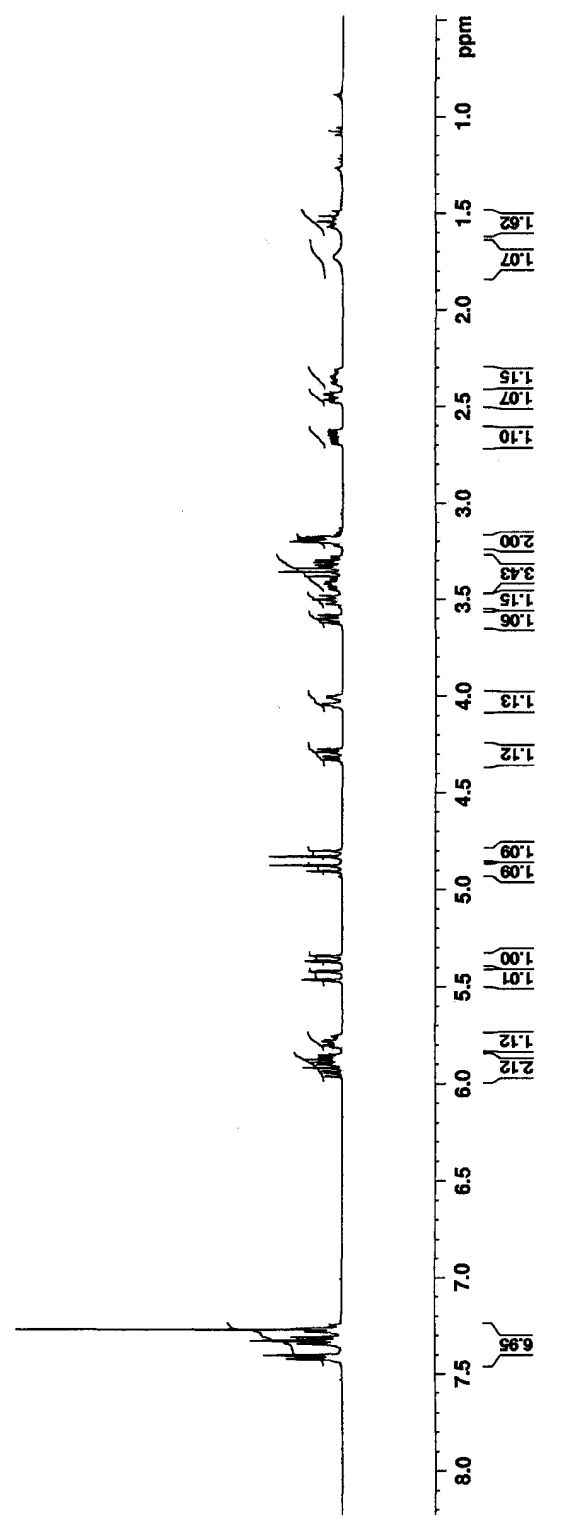



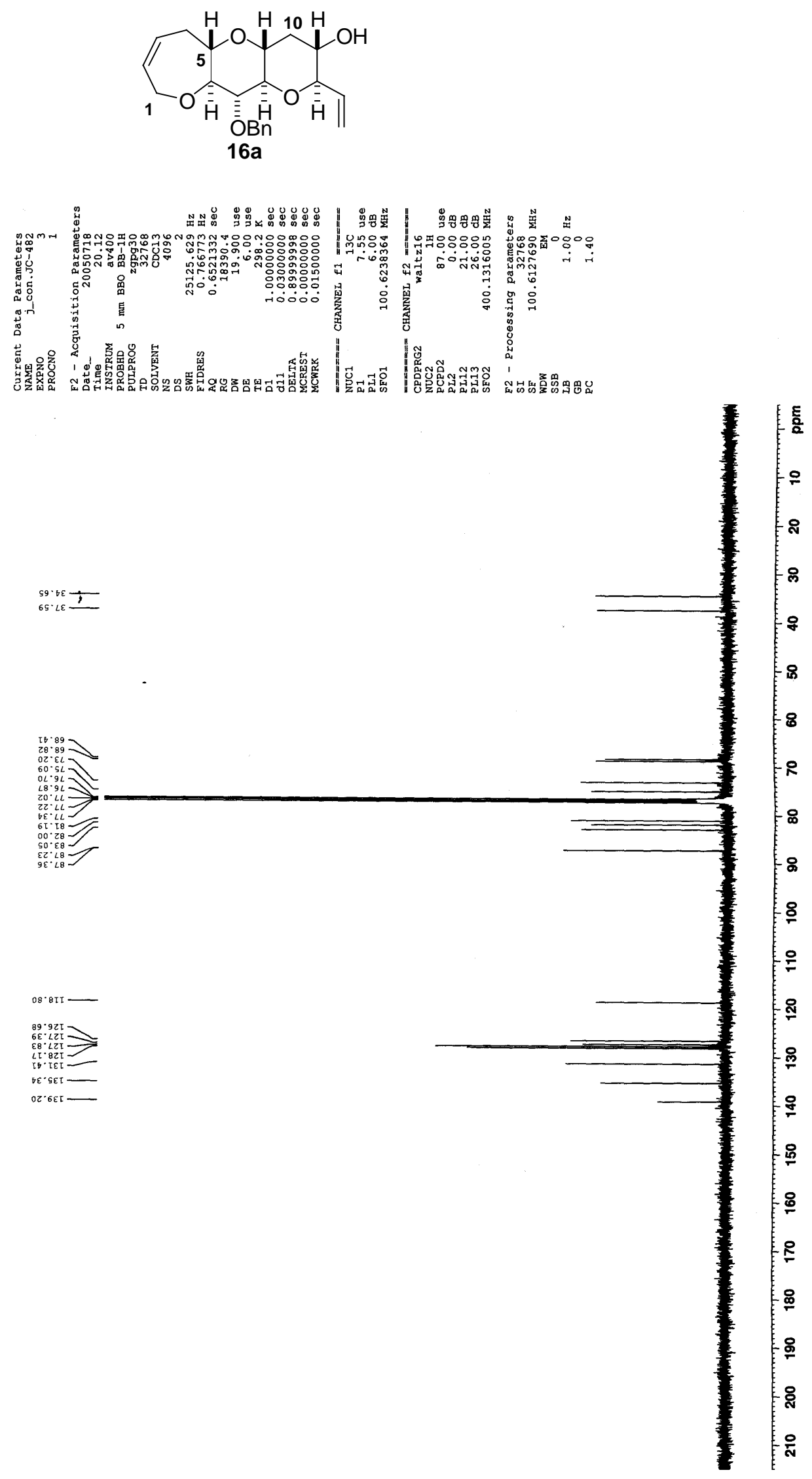

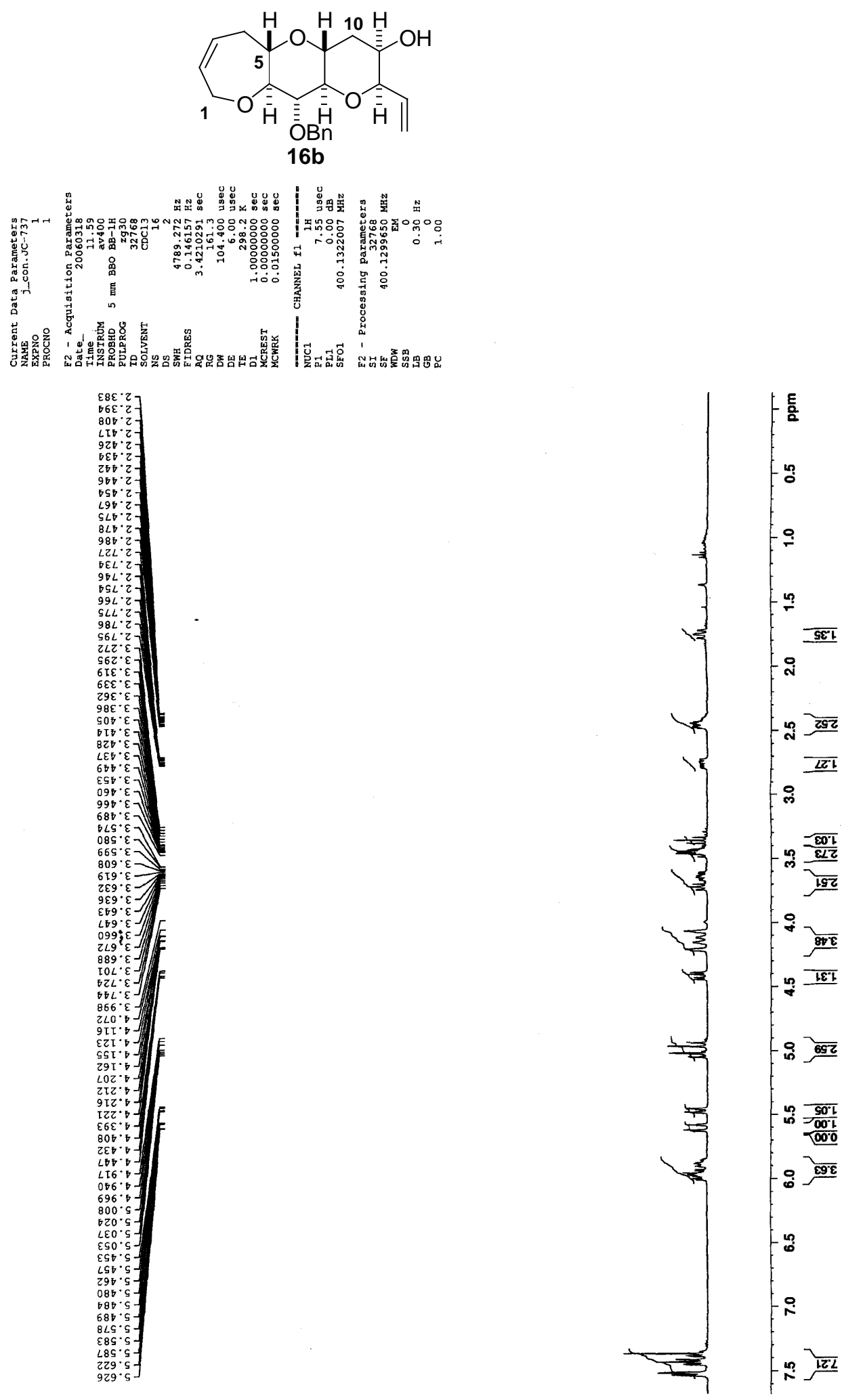

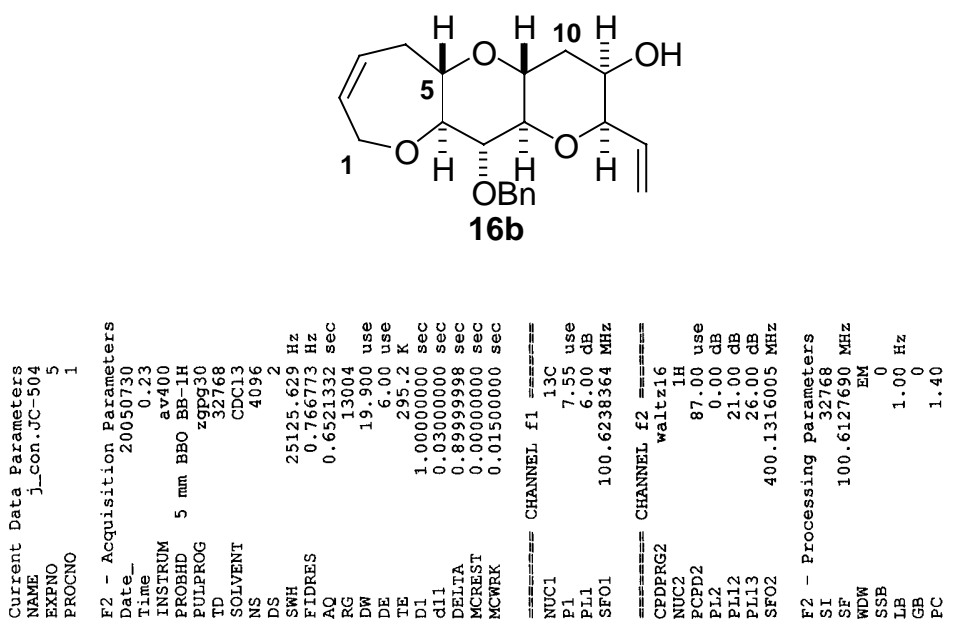

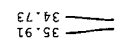
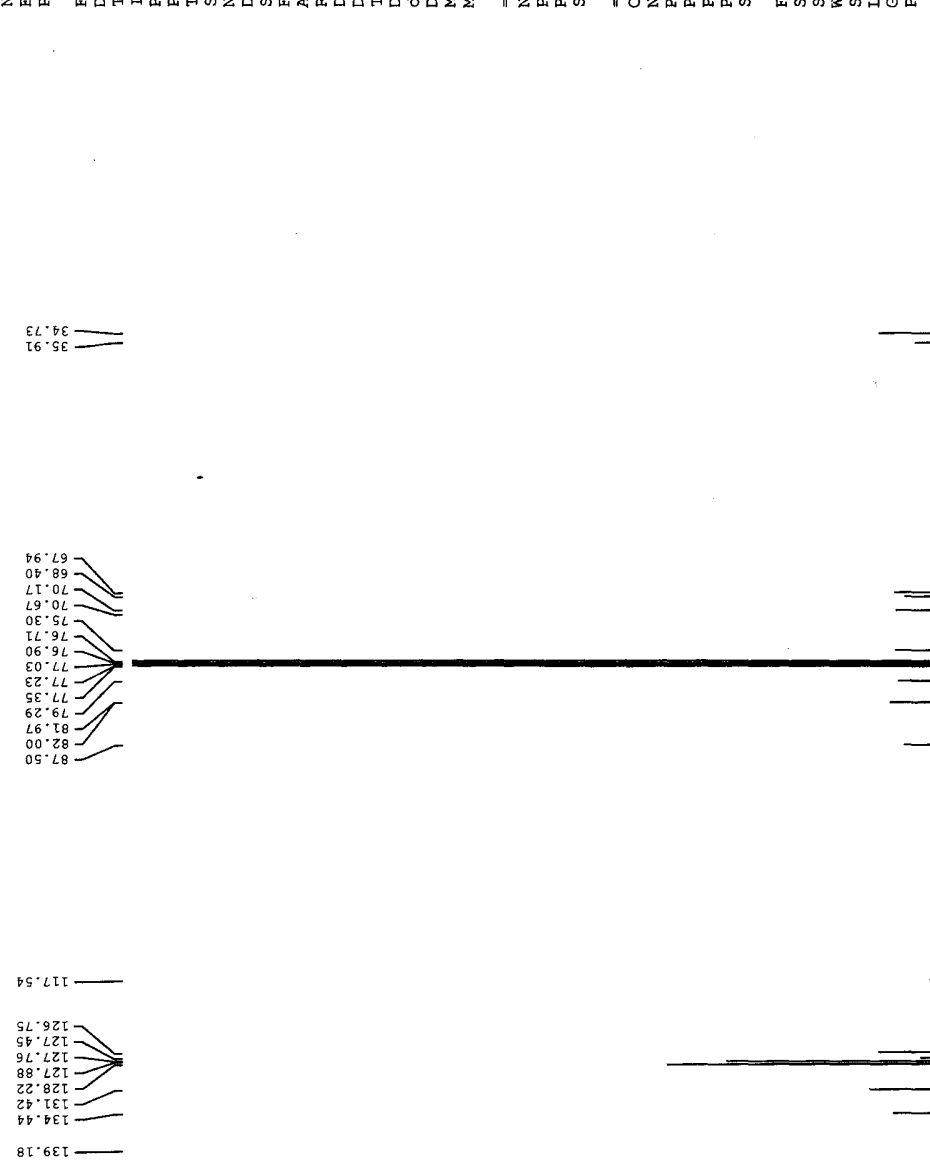

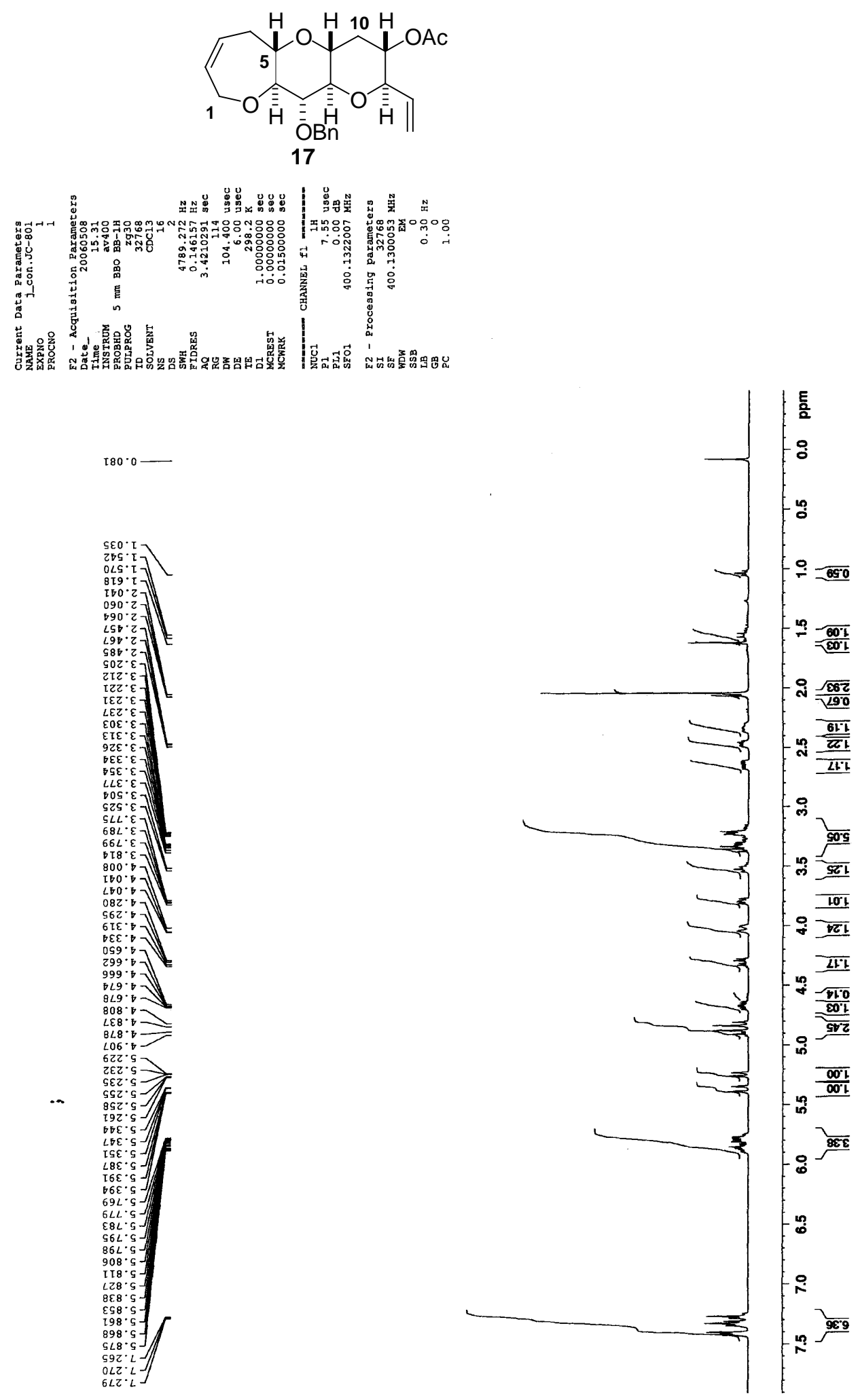

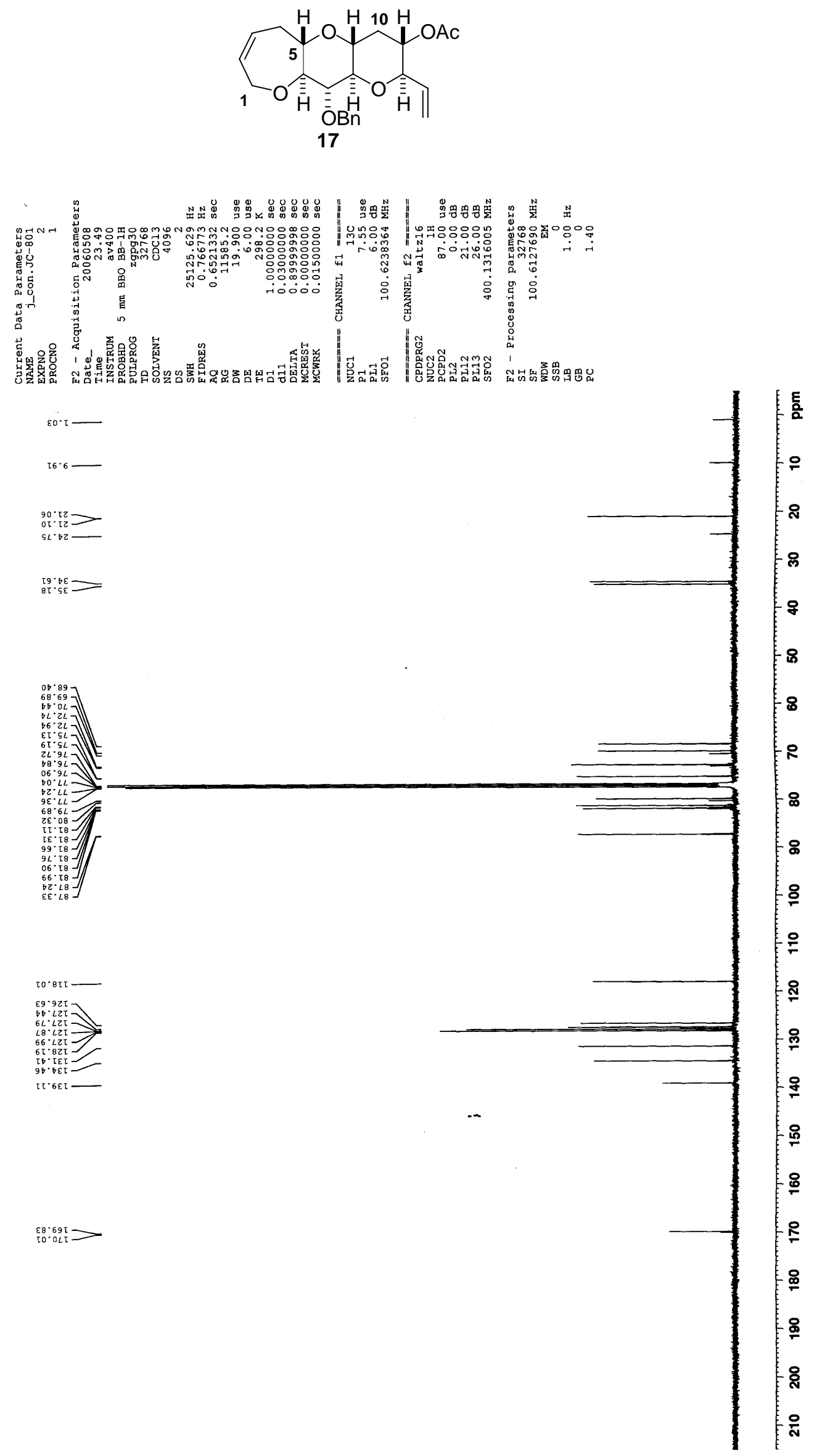


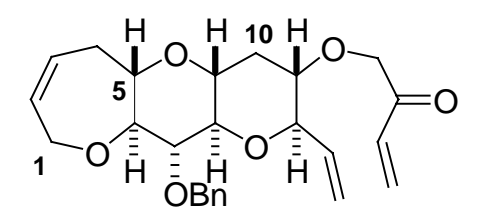

18
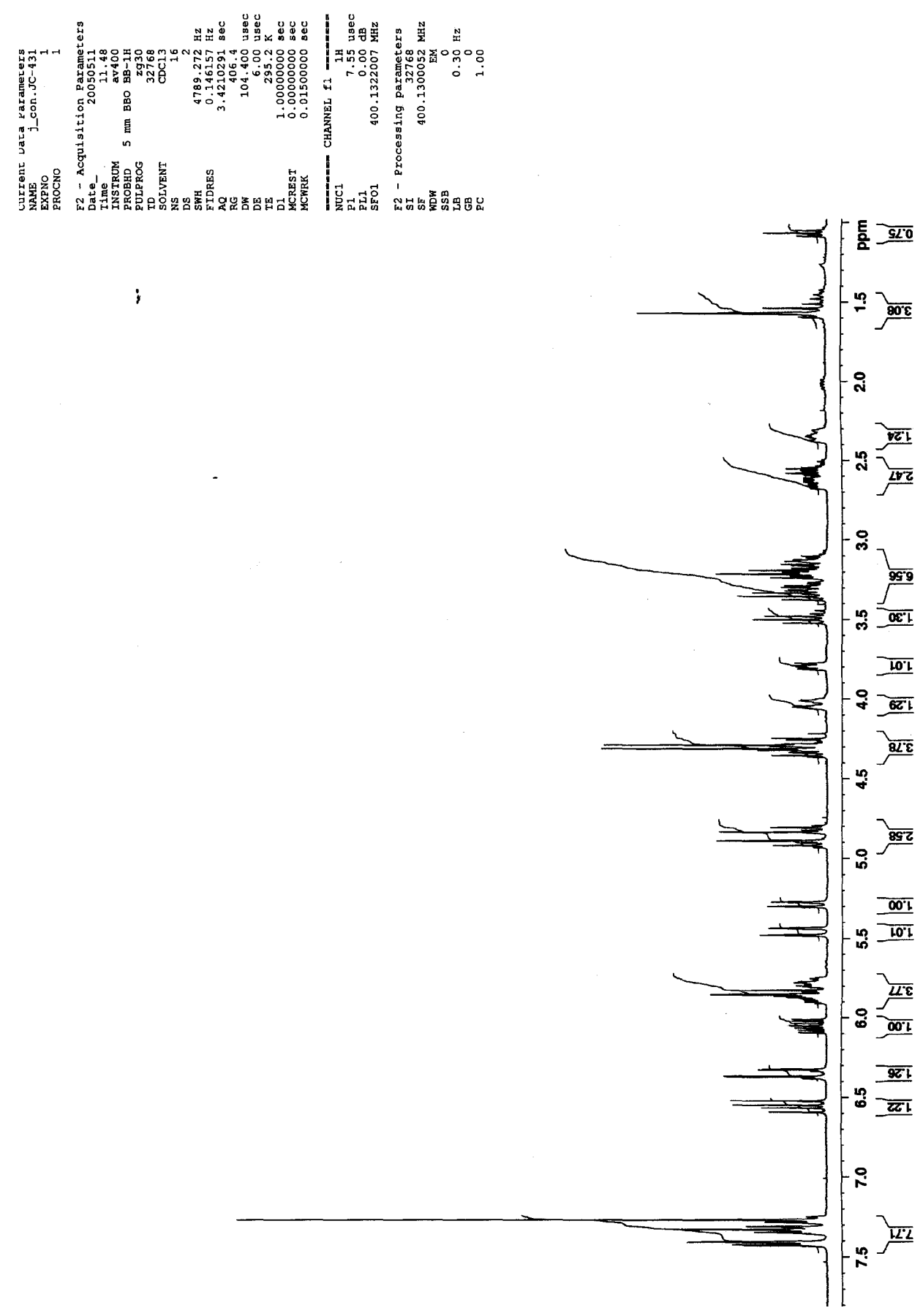


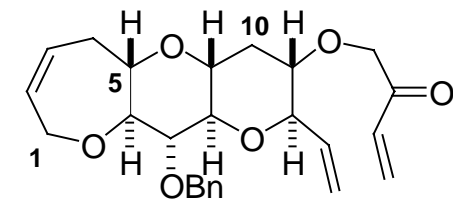

18
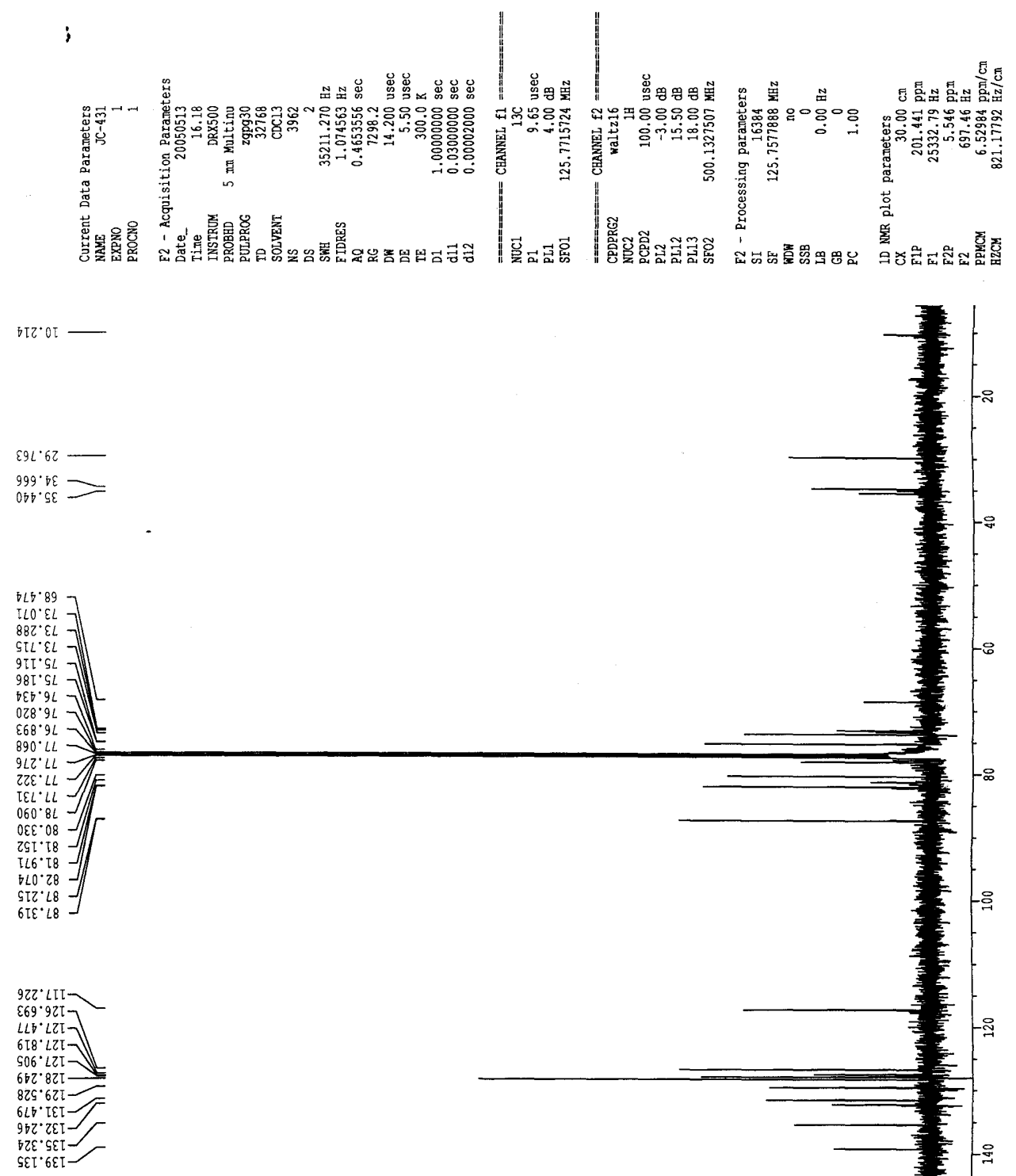

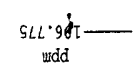

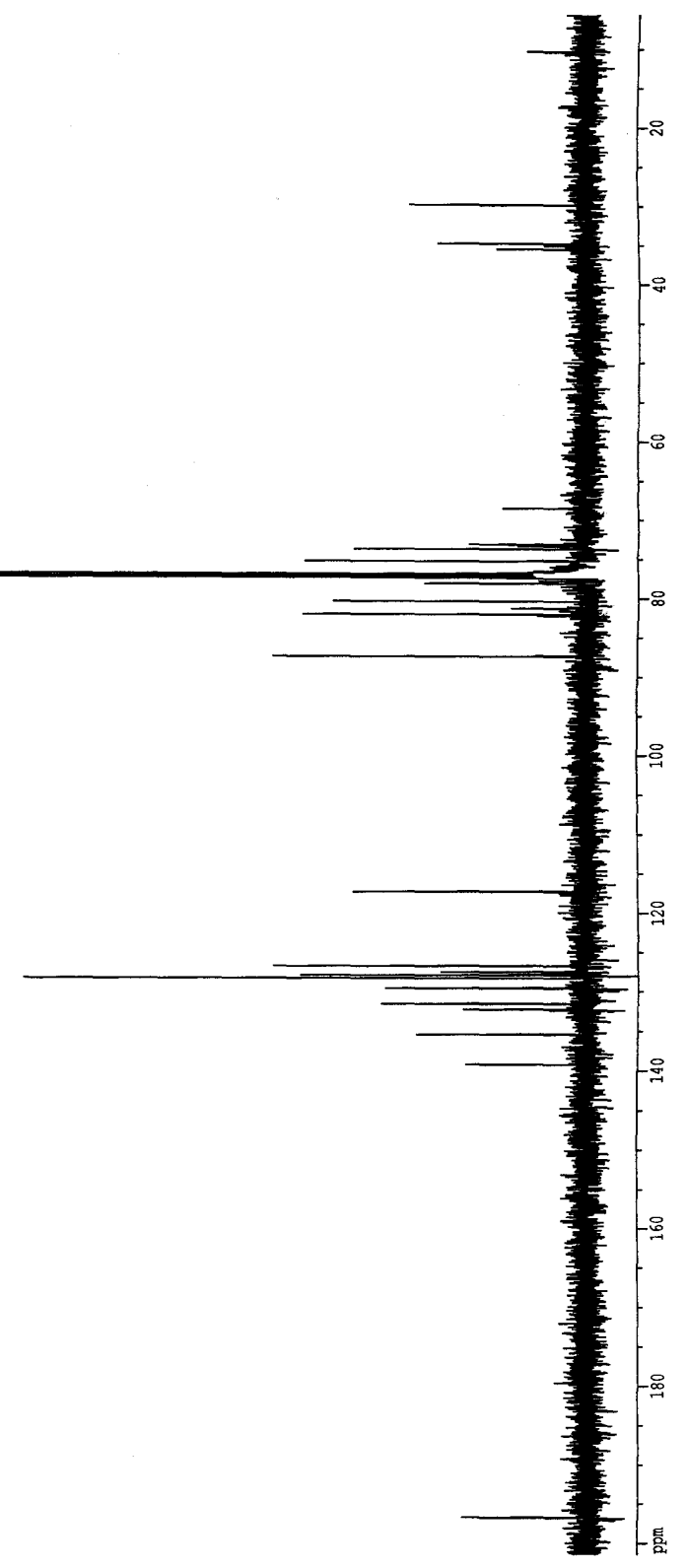



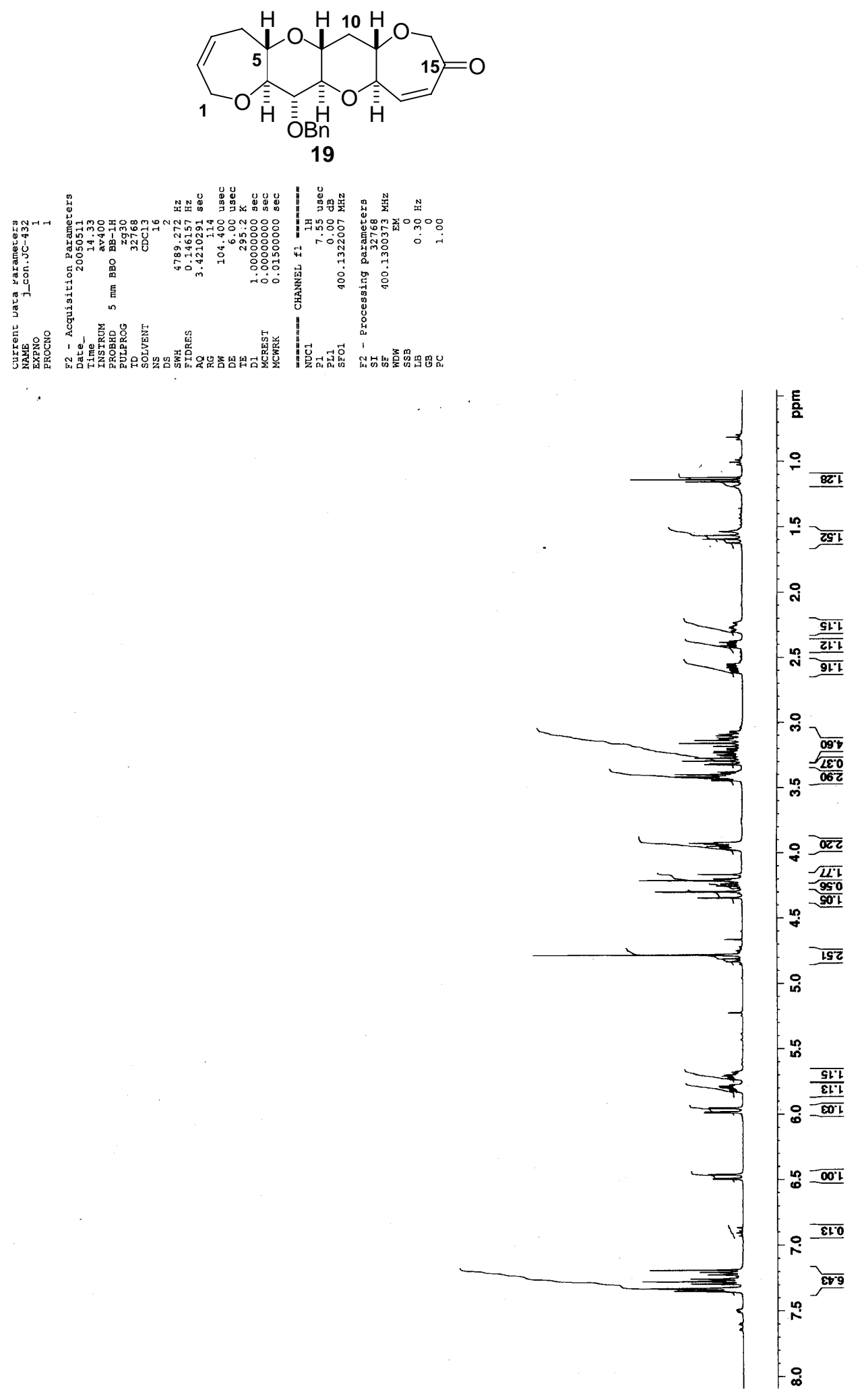


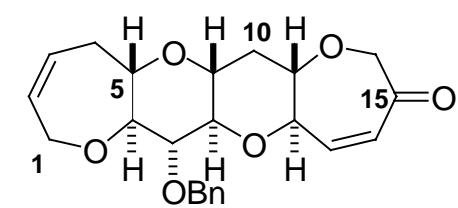

19

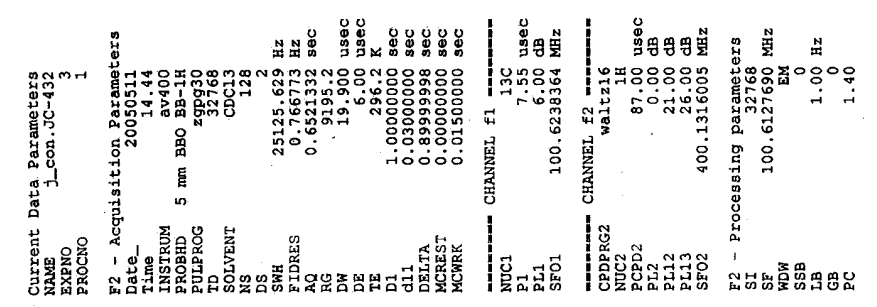

$o \varepsilon \cdot g I$

$85 \cdot b \varepsilon=$
$8 \tau \cdot 9 \varepsilon=$

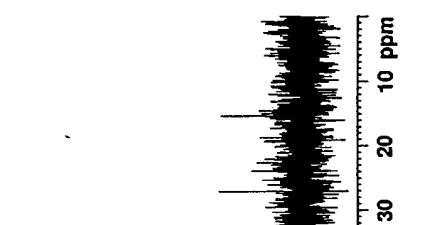

$66 \cdot 89$
$66.2 L$

SE. $s L>$

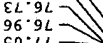

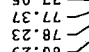

$62 \cdot 08$

$65^{\circ} \mathrm{LB}$
$80.28-$

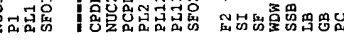



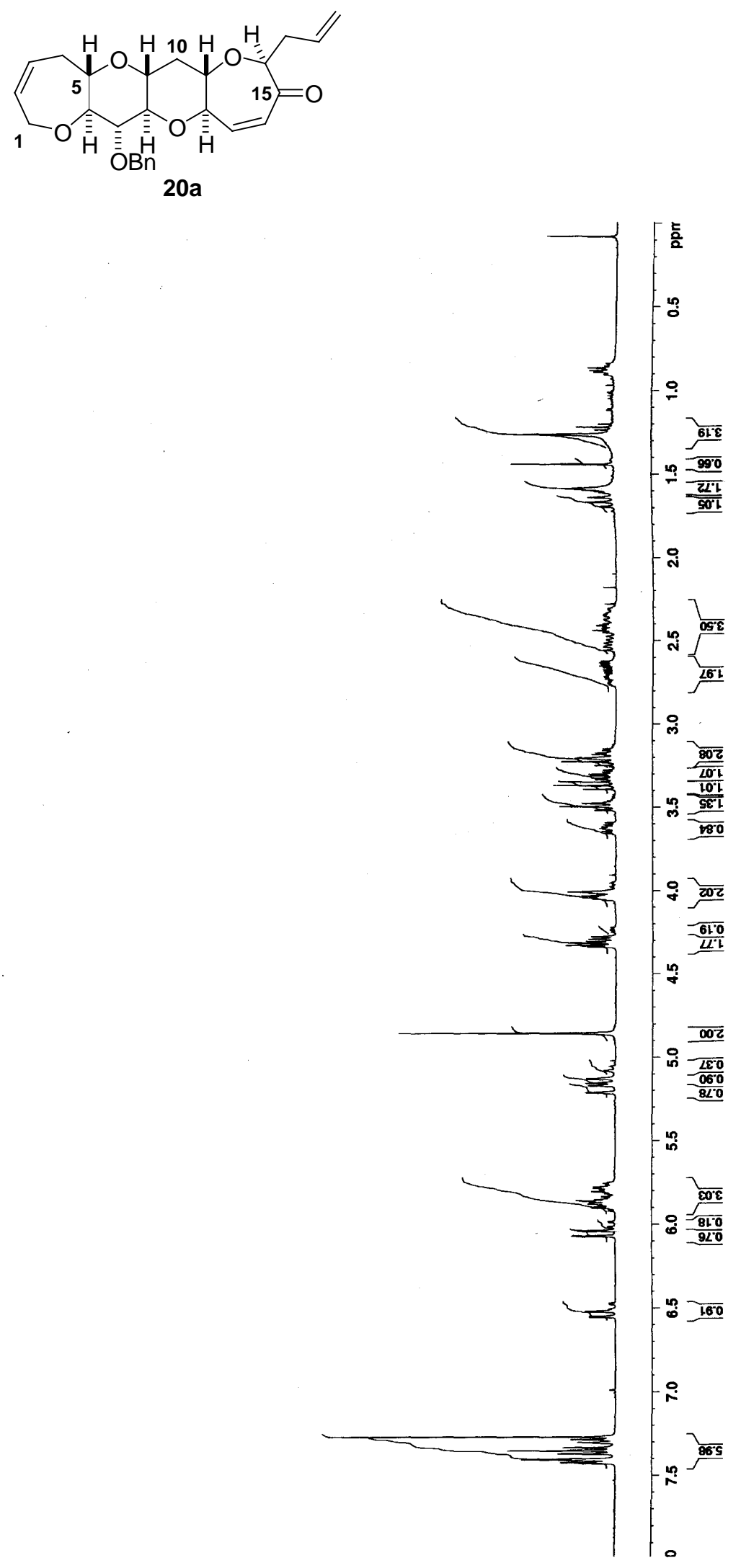

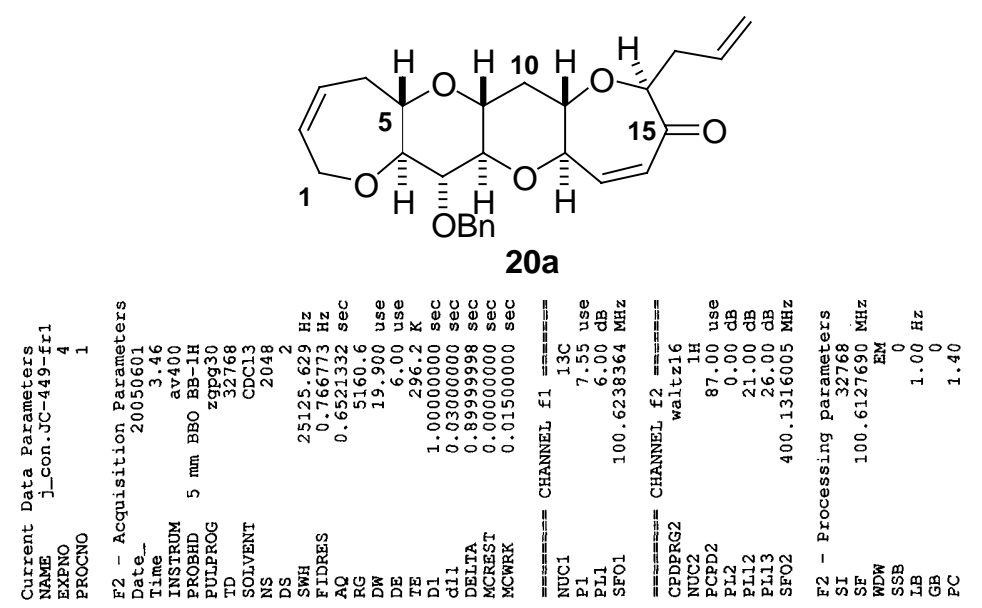

$\tau L^{\cdot 62}=$
$\varepsilon \varepsilon \cdot 0 \varepsilon=$
$8 \sigma^{\circ} \cdot 6 \varepsilon=$
$6 z \cdot 5 \varepsilon=$
$z Z^{-} 9 \varepsilon=$

E8. 102

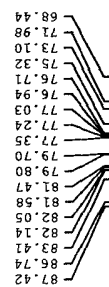

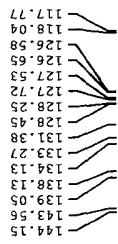

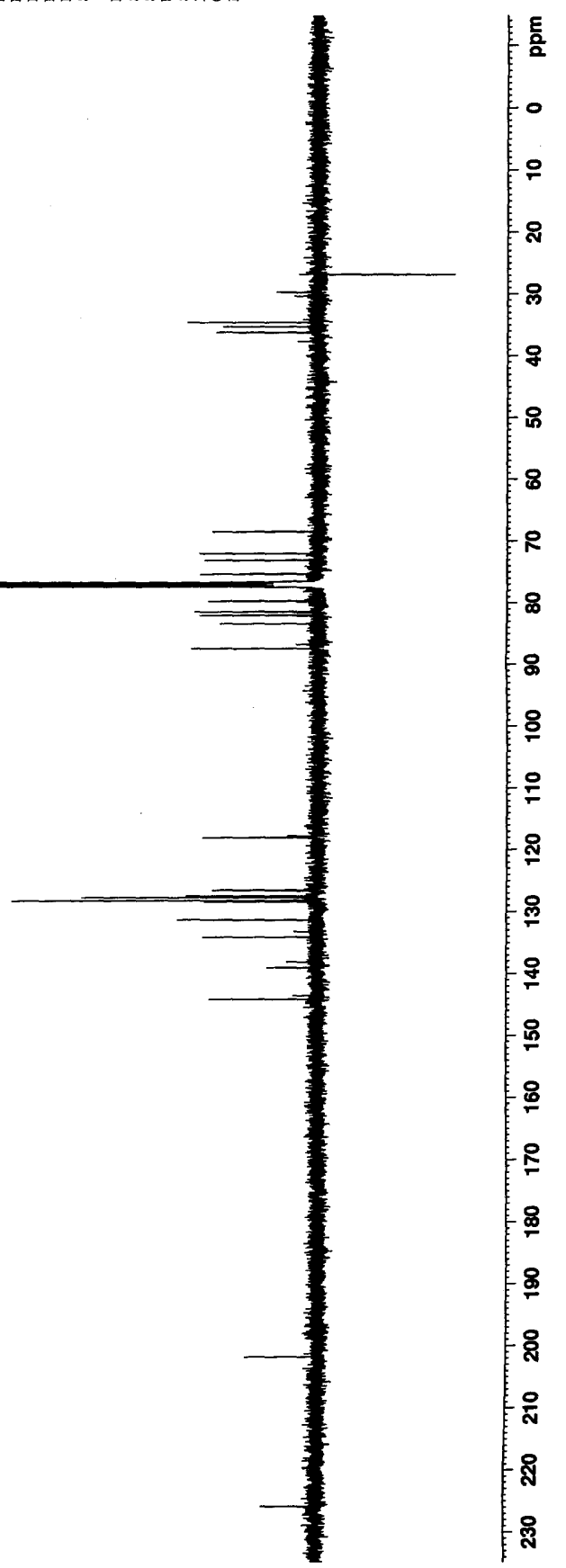



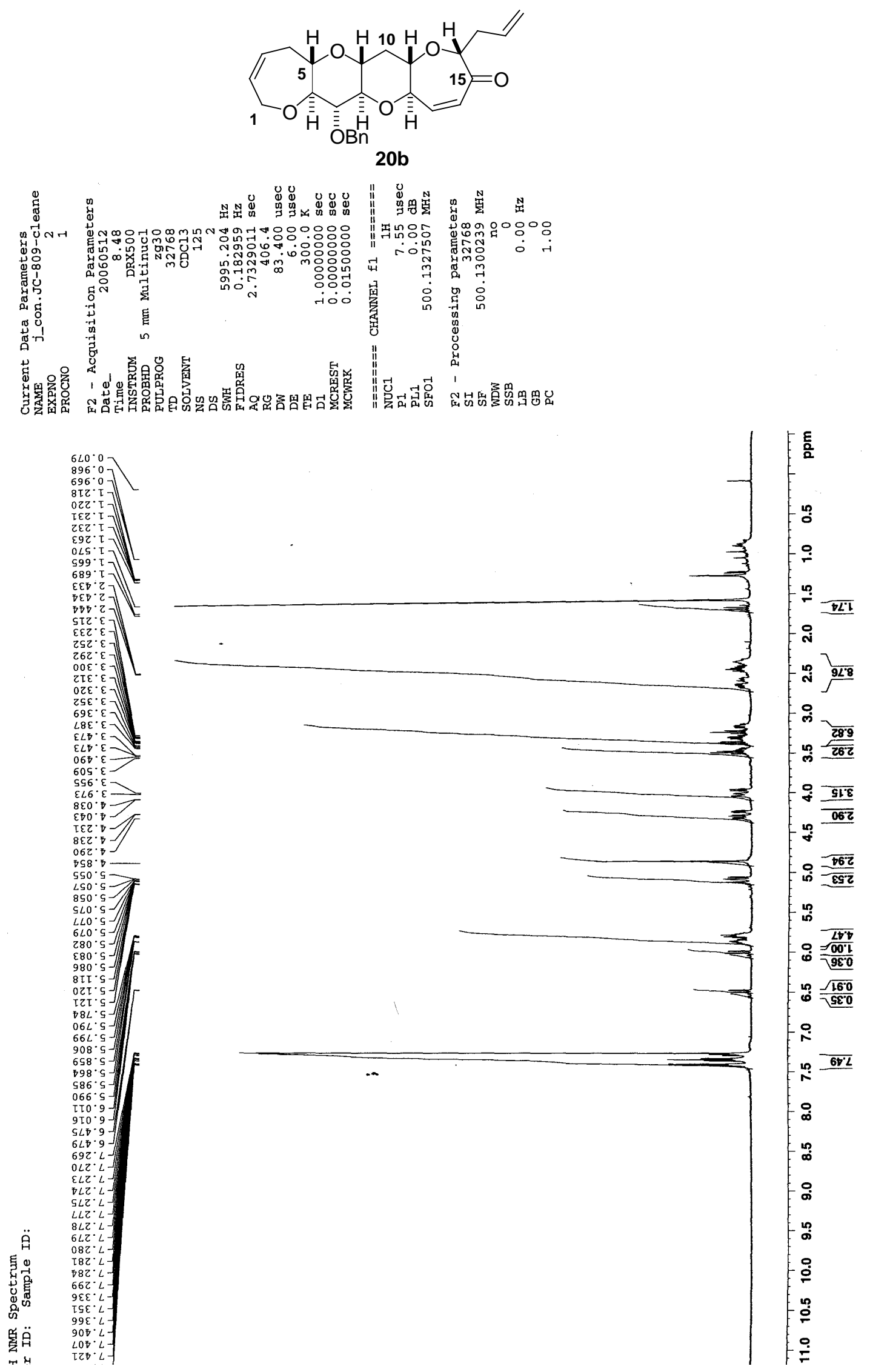

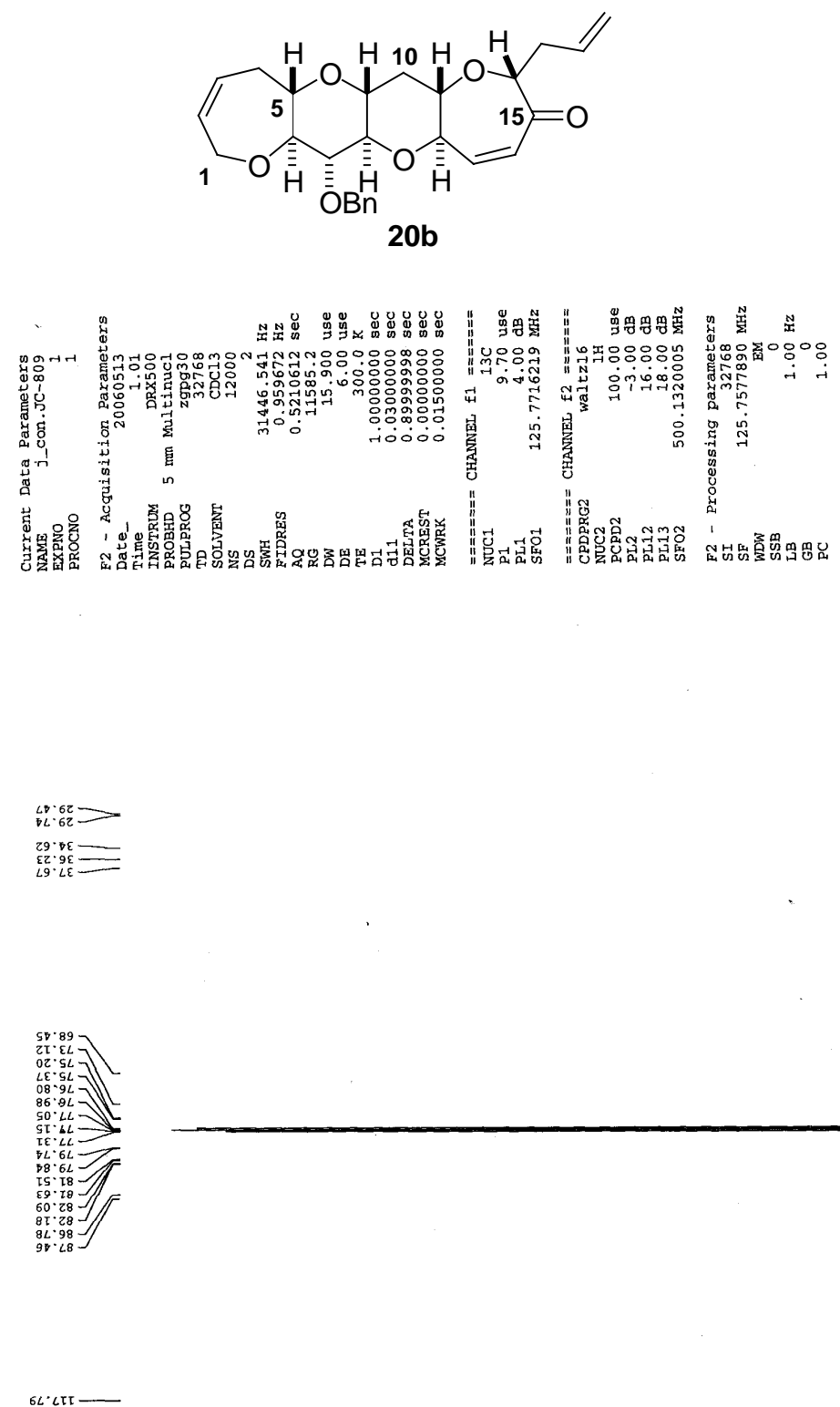

09.921

$99 \cdot 92 \mathrm{~T}$
$\nabla \mathrm{s}^{\circ} \mathrm{LZ \textrm {T }}$

$\$ L \cdot L z \tau$
$z z \cdot .8 z \tau=$
$8 z \cdot 8 z T$

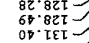

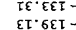

65. हD -

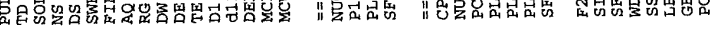

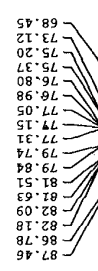

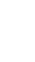

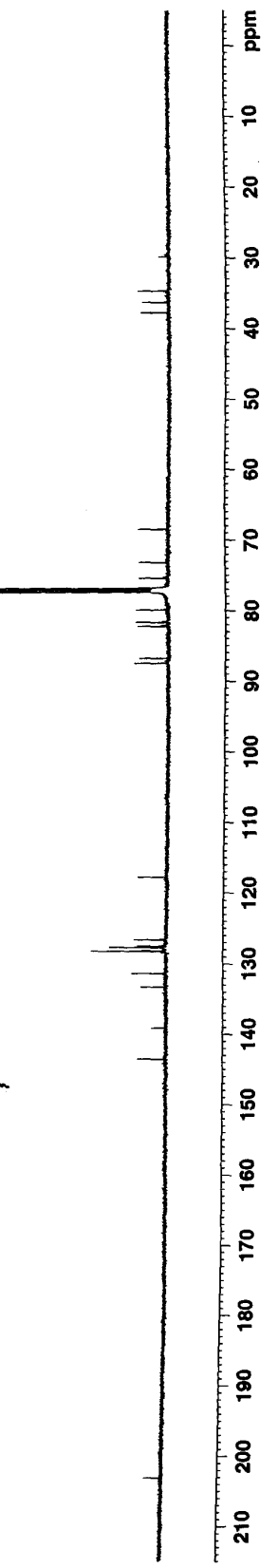



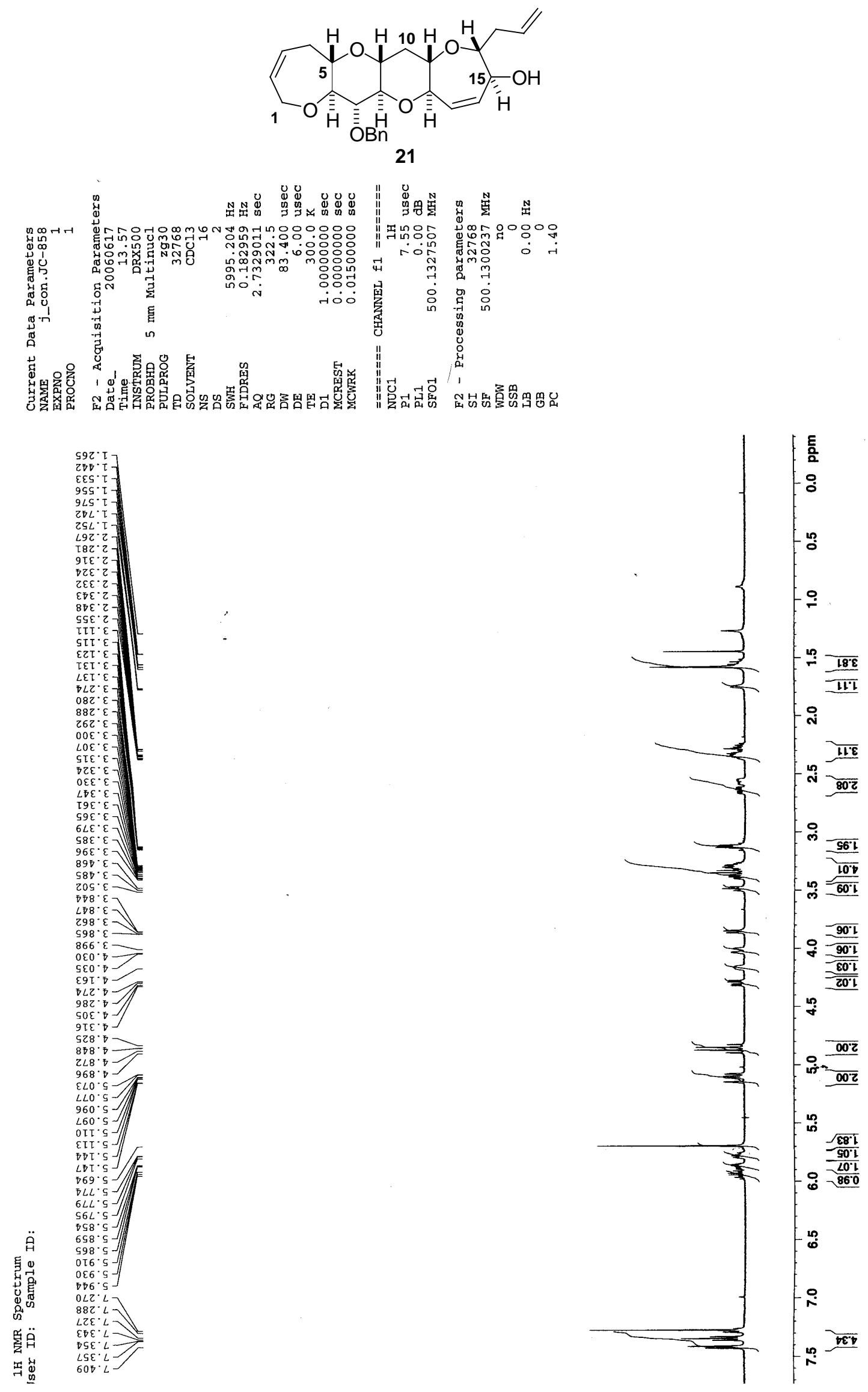

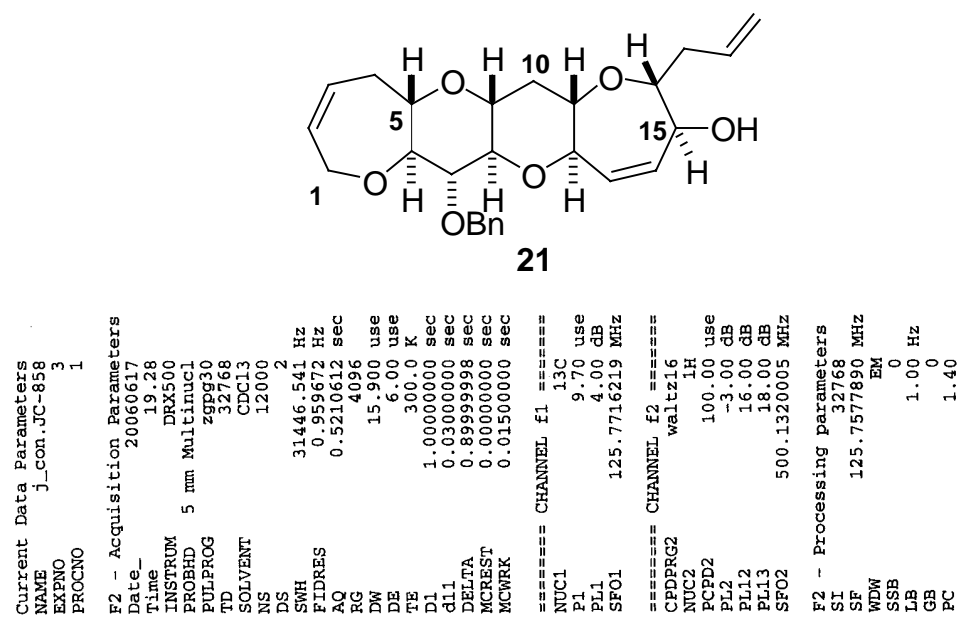

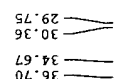

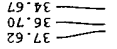

ZI. $\angle I T-$

95: $927 \mathrm{gzT}$

stivi

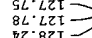

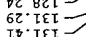

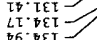
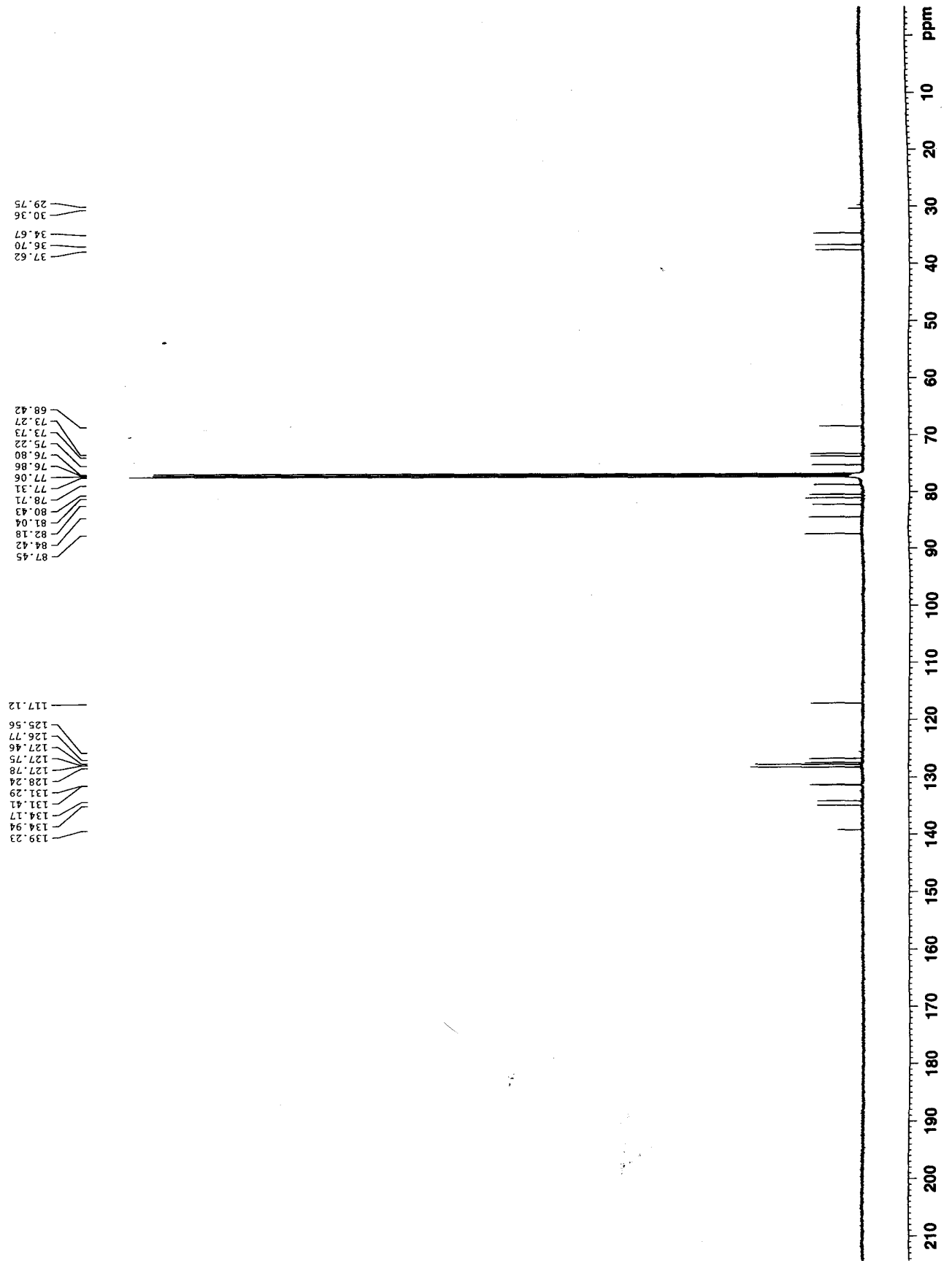

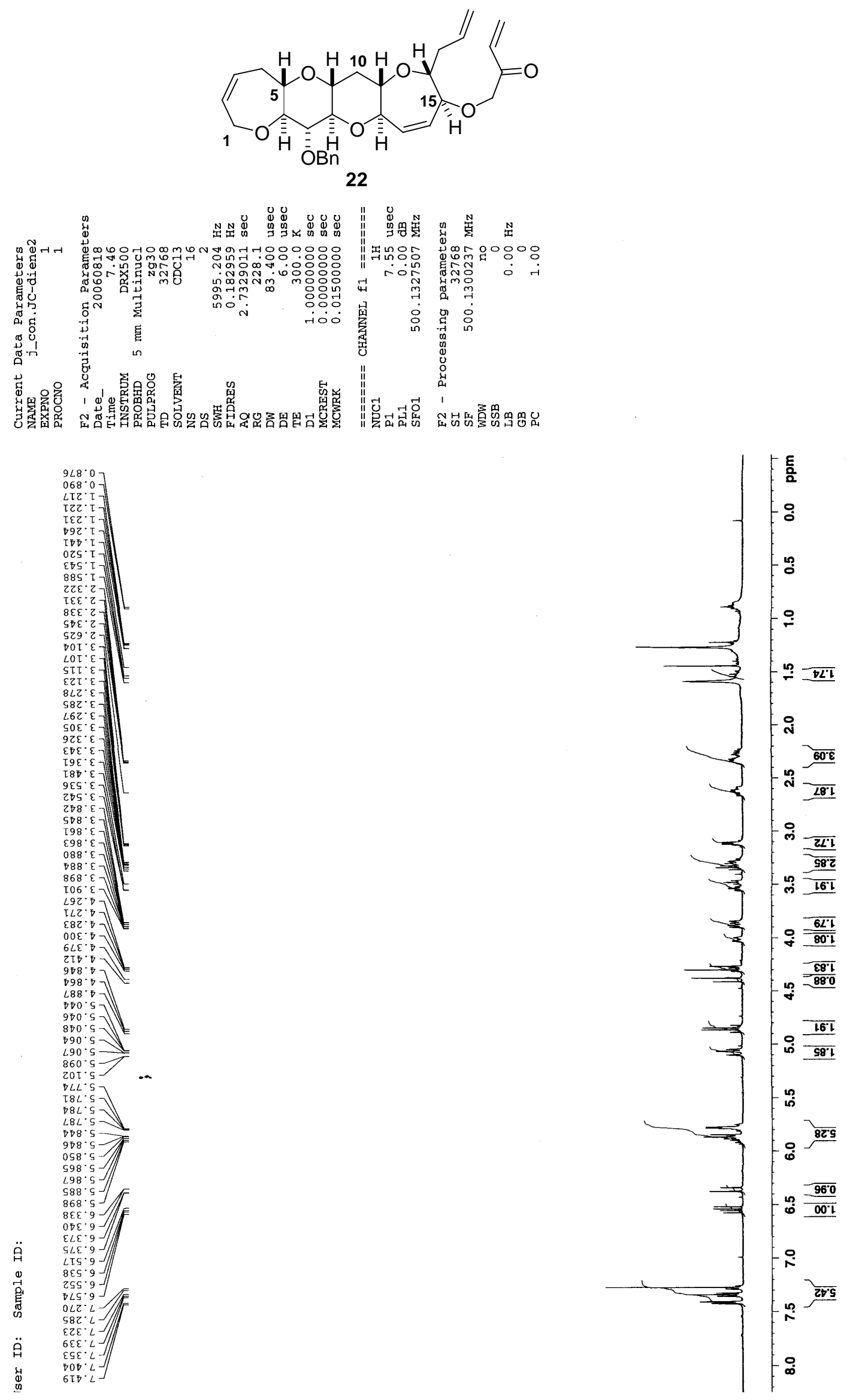

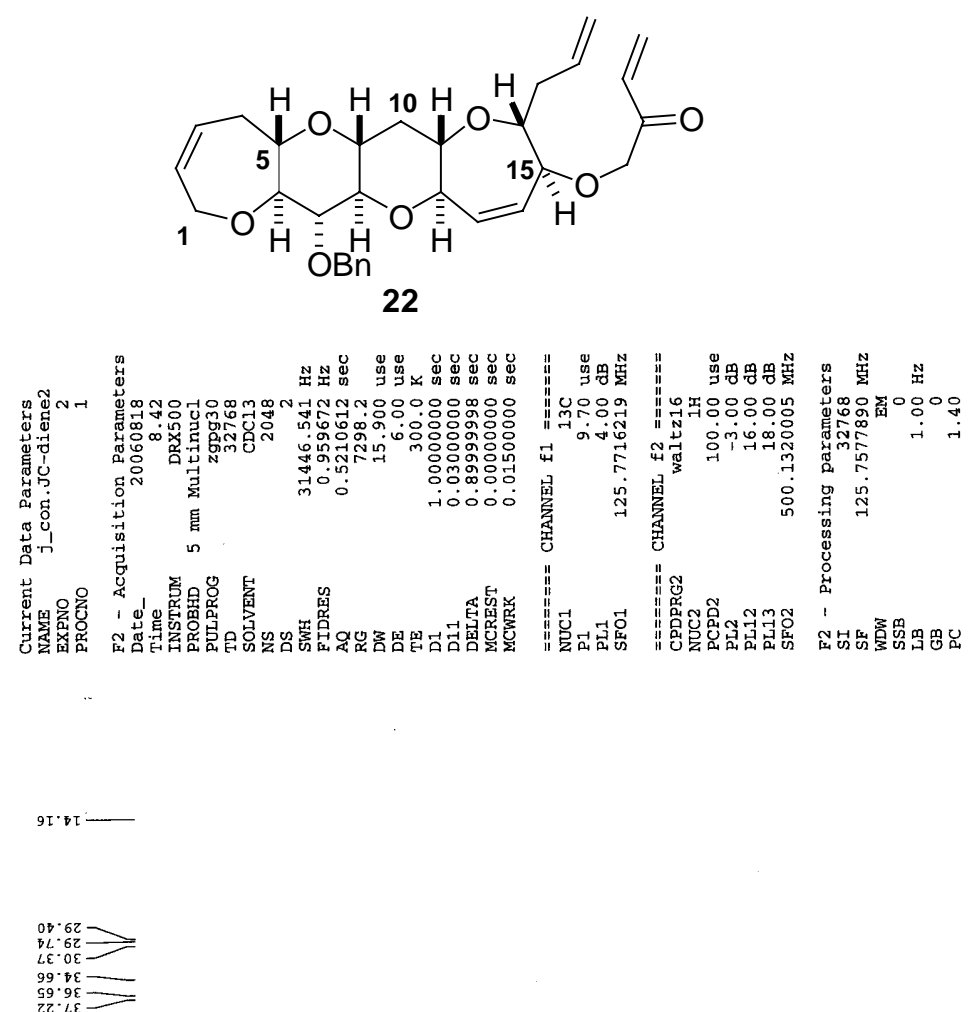

$99 \cdot \nabla \varepsilon=$
$59 \cdot 9 \varepsilon=$
$\mathrm{zZ} \cdot L \varepsilon=$

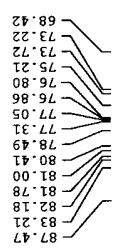

zo:LTr-

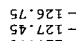

$8 L \cdot L Z \tau$
$\varepsilon z \cdot .8 z T=1$

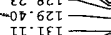

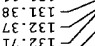

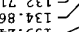
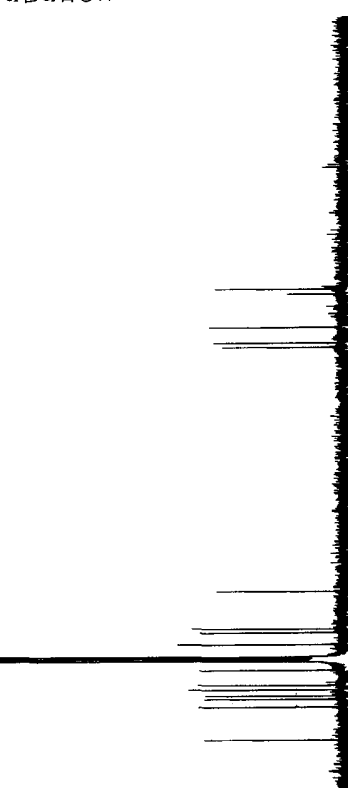


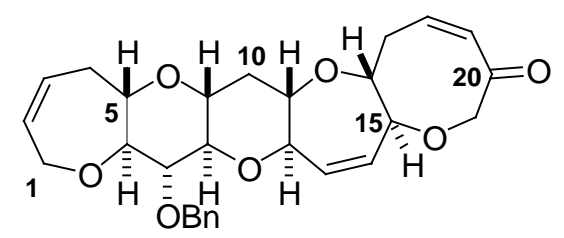

23
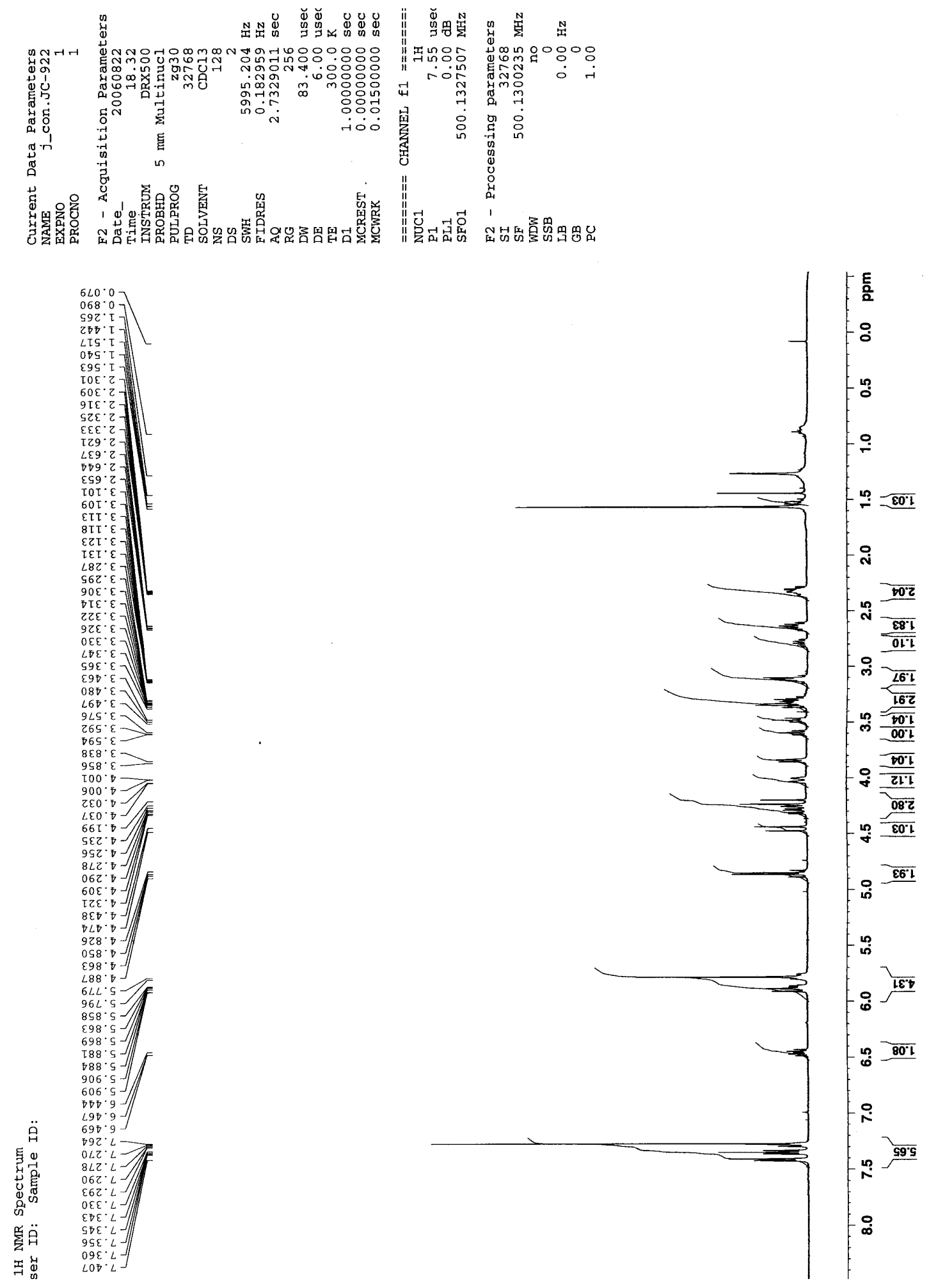

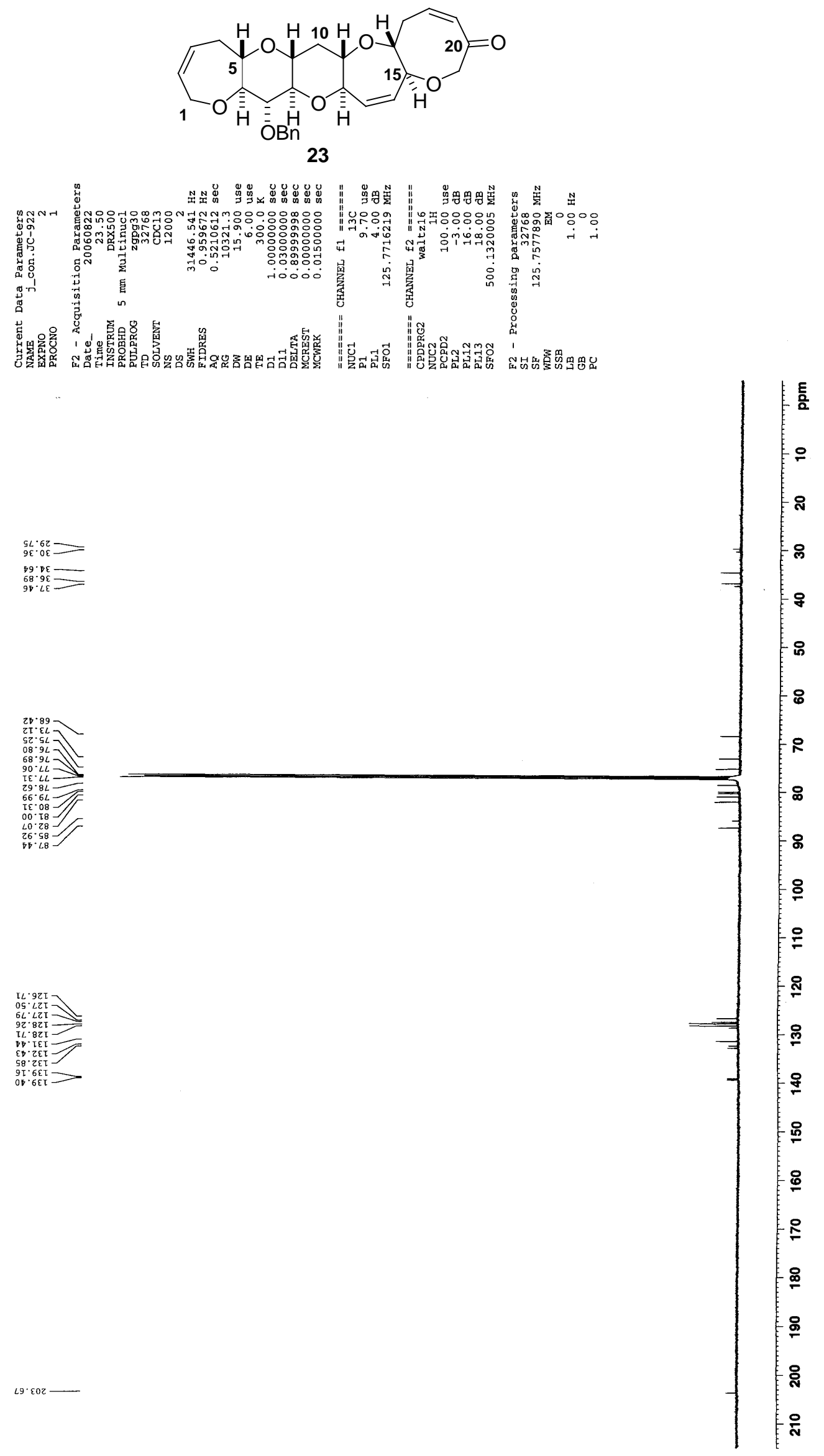